\title{
The stellar halo of the Galaxy
}

\section{Amina Helmi}

Received: 15 December 2007 / Published online: 22 April 2008

(C) The Author(s) 2008

\begin{abstract}
Stellar halos may hold some of the best preserved fossils of the formation history of galaxies. They are a natural product of the merging processes that probably take place during the assembly of a galaxy, and hence may well be the most ubiquitous component of galaxies, independently of their Hubble type. This review focuses on our current understanding of the spatial structure, the kinematics and chemistry of halo stars in the Milky Way. In recent years, we have experienced a change in paradigm thanks to the discovery of large amounts of substructure, especially in the outer halo. I discuss the implications of the currently available observational constraints and fold them into several possible formation scenarios. Unraveling the formation of the Galactic halo will be possible in the near future through a combination of large wide field photometric and spectroscopic surveys, and especially in the era of Gaia.
\end{abstract}

Keywords Galaxy: halo - Galaxy: formation - Galaxy: evolution - Galaxy: kinematics and dynamics

\section{Introduction}

A word of caution is necessary before we start our journey through the stellar halo of the Galaxy. While working on this article, I could not help but wonder whether this was a good time to write a review on the stellar halo (see, for example, previous excellent reviews by Gilmore et al. 1989; Majewski 1993; Bland-Hawthorn and Freeman 2000; Freeman and Bland-Hawthorn 2002). The advances in the recent past have been enormous thanks to many (ongoing) large wide field photometric and spectroscopic surveys. In the past 2 years, almost every month a new satellite galaxy or a new stream

\footnotetext{
A. Helmi ( $\square)$

Kapteyn Astronomical Institute, University of Groningen,

P.O. Box 800, 9700 AV Groningen, The Netherlands

e-mail: ahelmi@astro.rug.nl
} 
was discovered. This in itself may be a justification for writing a review, but it also may lead to an article that is rapidly out of date.

On the other hand, this of course, is only a sign of the field's health and its great promise for the young generations of scientists hoping to unravel the formation of the Galaxy. The reader is hereby warned that our understanding of the Galactic halo is still evolving significantly. In an attempt to avoid the (almost unavoidable) risk of becoming quickly outdated, the author has decided to review those properties of the stellar halo that (hopefully) provide the most direct clues to its evolutionary path.

The stellar halo is arguably the component that contains the most useful information about the evolutionary history of the Galaxy. This is because the most metal-poor stars in the Galaxy and possibly some of the oldest ones are found here. It therefore provides us with a picture of the Milky Way in its early stages of evolution. Low-mass stars live for much longer than the present age of the Universe, and so retain in their atmospheres a record of the chemical elements of the environment in which they were born. The very metal-poor halo stars are thus fossils, whose chemical abundance and motions contain information of their sites of origin. As Eggen et al. (1962) nicely put it: "... a study of these subsystems allows us partially to reconstruct the Galactic past because the time required for stars in the Galactic system to exchange their energies and momenta is very long compared with the age of the Galaxy. Hence knowledge of the present energy and momenta of individual objects tells us something of the initial dynamic conditions under which they were formed".

Despite its crucial importance, our knowledge of the stellar halo is fragmentary. This is at least partly driven by the paucity of halo stars (near the Sun roughly only one star in a thousand belongs to the halo, as discussed in Sect. 2.2). This implies that generally a set of suitable (and preferably unbiased) selection criteria are used to find these precious fossils, as we shall see later in this review.

The study of the formation of the Galaxy is particularly relevant at this point in time. There is a cosmological framework in place, the $\Lambda$ cold dark matter (CDM) paradigm, which broadly describes from first principles how structures in the Universe have evolved (e.g. Springel et al. 2006 and references therein). In this model, galaxies form hierarchically, through the amalgamation of smaller proto-systems. Despite the many successes of this theory, particularly on large scales, we do not yet understand in detail how galaxies form. Many of the shortcomings of this model have become evident through comparisons to the properties of our Galaxy and its nearest neighbors (e.g. the "missing satellites" problem, Klypin et al. 1999; Moore et al. 1999). Therefore, it becomes clear that studies of the Galaxy and its components are crucial for a proper and complete understanding of galaxy evolution in the Universe.

This review describes the various characteristics of the stellar halo in the hope that these will aid us in our understanding of the formation of the Galaxy. We start by discussing the spatial structure and global kinematics in Sect. 2. This approach is rather classical, since we shall discuss separately the presence of substructure in Sect. 3. In principle, substructure can strongly affect the determination of the density profile or of the shape of the stellar halo, e.g. by introducing overdensities which may not always be easy to identify as such (e.g. Newberg and Yanny 2006; Xu et al. 2007; Bell et al. 2007). Given our current understanding of the Galactic halo, such substructures may well dominate at large radii, but at small distances (i.e. the stellar halo near and interior 
to the Solar circle), the mixing timescales are sufficiently short that even if all of the stellar halo had been formed through a sequence of dissipationless mergers it would be completely smooth spatially (see Helmi and White 1999).

In Sect. 4, we describe our current knowledge of the metallicity distribution of halo stars, and particularly focus on what it reveals about star formation in the early Universe. We also discuss what chemical abundance patterns tell us about the process of formation of the Galaxy. Not only halo field stars are interesting, but also globular clusters and satellite galaxies give us insight into the characteristics and evolution of the Milky Way halo. These are discussed in Sect. 5.

In Sect. 6, we discuss formation scenarios for the stellar halo taking into account most (if not all) observational constraints presented in earlier sections. Recent technological advances have allowed us to begin to probe halos in other galaxies. Their characteristics and a comparison to our own stellar halo are briefly mentioned in Sect. 7. Finally, we provide a brief summary and outlook in Sect. 8.

\section{Spatial structure and global kinematics}

\subsection{Star counts: density profile and shape}

Star counts have been used in astronomy for over a century to derive the structure of our Galaxy (and even of the Universe, e.g. Kapteyn and van Rhijn 1920; Shapley 1918). They have led to the conclusion that our Galaxy has several physically distinct components, namely the thin and thick disk, the stellar halo and the bulge/bar (e.g. Bahcall and Soneira 1980; Gilmore and Reid 1983).

The structure of the stellar halo is intimately linked to how the Galaxy formed (Freeman and Bland-Hawthorn 2002). Different scenarios predict different shapes and correlations with properties such as age, metallicity, etc., as we shall discuss later. In the simplest case, the structure of the stellar halo could also give us insight into the structure of the dark matter halo. For example, if a significant fraction of the dark matter had been baryonic (composed by MACHOs, Paczynski 1986), then the stellar and dark halos would presumably be indistinguishable. However, this scenario appears unlikely, as the microlensing surveys are unable to assign more than $8 \%$ of the matter to compact dark objects (Tisserand et al. 2007; although see Alcock et al. 2000b for a different while earlier result).

In modern cosmological models (namely, cold dark matter dominated), it is now clear that the structure of the stellar and dark halo do not necessarily follow one another. The dark halo is much more extended, and probably follows an NFW profile (Navarro et al. 1996). Its half-mass radius lies around $150 \mathrm{kpc}$ (Klypin et al. 2002; Battaglia et al. 2005, 2006), and therefore its structure is probably related to the present-day nearby large scale tidal field (Navarro et al. 2004). On the other hand, the stellar halo is highly concentrated (e.g. the effective radius as traced by the halo globular clusters is well within the Solar circle, Frenk and White 1982), a property that likely reflects a very early formation epoch ${ }^{1}$ (Moore 2001; Helmi et al. 2002).

\footnotetext{
1 This refers to the inner stellar halo; as we shall show in Sect. 3 it is clear that the outer stellar halo is still forming.
} 
In the early years, star counts were based on photographic images, and were usually restricted to just a few fields. This was also the basis for the pioneering Bahcall and Soneira (1980) model of the Galaxy. These authors took the approach that our Galaxy is "just" like any other galaxy, and so it should be possible to reproduce the star counts using models that fit the light distribution of external galaxies. For example, they proposed the halo followed a "de Vaucouleurs" spherical profile, which is characteristic for elliptical galaxies (although it is now known that not all elliptical galaxies are well-fit by this function; furthermore Morrison (1993) showed that a power-law $\rho \propto r^{n}$ with $n \sim-3.5$ as proposed by Harris (1976) on the basis of halo globular clusters, provides a better fit for the stellar halo than an $R^{1 / 4}$ law).

Already in early studies (Kinman et al. 1966) there was evidence that the stellar halo's shape varied with distance from the Galactic center. These studies favored a halo that was rounder in the outskirts (minor-to-major axis ratio $q \sim 1$ ) and more flattened in the inner parts $(q \sim 0.5$; Schmidt 1956; Hartwick 1987; Preston et al. 1991). Typically RR Lyrae and blue horizontal branch (BHB) stars were used as tracers. Even today these stars are commonly used to isolate samples of true halo stars. Such stars are only found in old populations, and they are relatively bright so they can be observed with modest telescopes at distances as large as $100 \mathrm{kpc}$ from the Galactic center (e.g. Clewley et al. 2005).

The density profile of the stellar halo is often parametrized in a principal axis cartesian coordinate system as

$$
\rho(x, y, z)=\rho_{0} \frac{\left(x^{2}+\frac{y^{2}}{p^{2}}+\frac{z^{2}}{q^{2}}+a^{2}\right)^{n}}{r_{0}^{n}}
$$

where $n$ is the power-law exponent (and because of the finite extent of the Galaxy, it is negative), and $q$ and $p$ are the minor- and intermediate-to-major axis ratios (in the axisymmetric case, $p=1$ ). The scale radius $a$ is often neglected because of the rather concentrated nature of the stellar halo (although see for example Phleps et al. 2005; Bica et al. 2006). Finally, $\rho_{0}$ is the stellar halo density at a given radius $r_{0}$, which is generally taken to be the solar radius for obvious reasons (and referred to as the local normalization). As we shall see below, this parametrization is likely to be an oversimplification because the shape as well as the exponent seem to vary with distance from the Galactic center.

\subsubsection{Modern pencil beam surveys}

Robin et al. (2000) have used a large combined set of deep star counts at high and intermediate galactic latitudes, and found a power-law index $n=-2.44$ and flattening $q=0.76$. Siegel et al. (2002) observed seven Kapteyn selected areas $\left(14.9^{\circ 2}\right)$ (hence ensuring uniform photometry) and preferred $n=-2.75$ and $q=0.6$. There are many delicate points in these analyses. For example, the counts also include stars from the thin and thick disks, which implies that the fits should also allow for the (self-consistent) modeling of these components (see top panels in Fig. 1). Furthermore there is a 

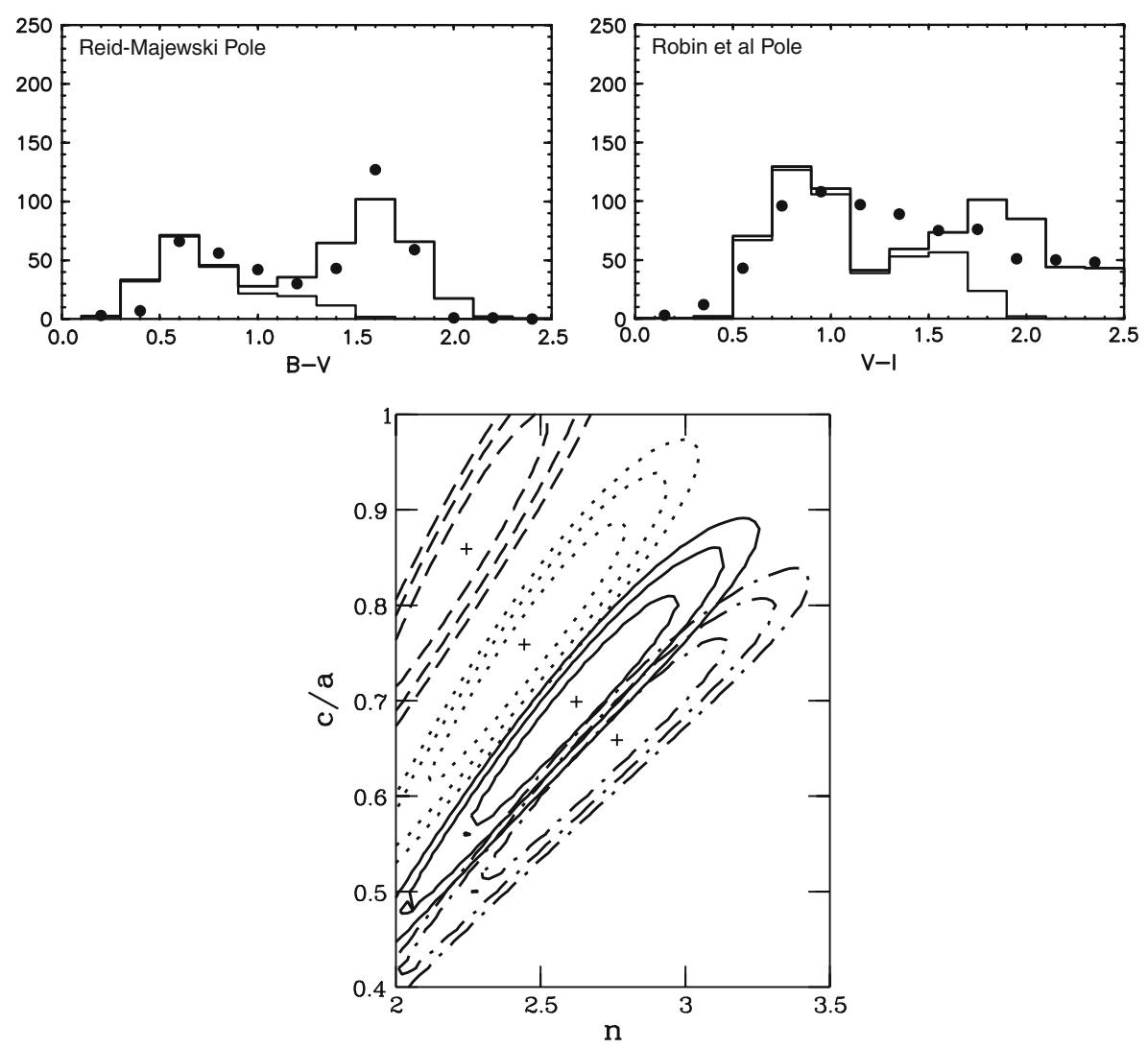

Fig. 1 Top panels: Color distributions of two fields used by Robin et al. (2000) to constrain the shape of the stellar halo. The dots correspond to the true number of observed stars in each color bin, while the heavy solid lines are the number of stars predicted by the model assuming a power law index of -2.44 , a flattening of 0.76 and a local density of $0.75 \times \rho_{0}$ for the stellar halo. Thin lines show the contribution of the halo alone. The red peak corresponds to nearby $\mathrm{M}$ dwarfs, while the blue peak indicates the location of the halo and thick disk turn-off points. Bottom panel: Iso-likelihood contours at 1, 2 and 3 sigmas in the plane (power law index, flattening) of the spheroid population, coded by the local density, going from 1 (solid), 0.75 (dotted); 0.50 (dashed) and 1.25 (dash-dotted) $\times \rho_{0}$. This diagram evidences the strong covariance between the shape and power-law index of the stellar halo. (From Robin et al. 2000. Courtesy of Annie Robin. Reproduced with permission of A\&A)

degeneracy in the modeling of the stellar halo in the sense that a less flattened halo requires a steeper density profile (bottom panel of Fig. 1). However, it is worth bearing in mind that in both studies the fits obtained are not perfect, and systematic differences between models and data are apparent, especially at the faint end (see also Fig. 6 in Robin et al. 2007). In particular, there are more stars towards the anticenter than predicted, and there are less stars observed towards the center. This is similar to what Morrison et al. (2000) find using turn-off halo stars (i.e. those with $(B-V) \sim 0.38$ ), who prefer $n=-3$ and $q=0.6$. These authors note that a two-component halo is unlikely to solve these problems, because it is incapable of enhancing the number counts towards the anticenter (a rounder halo would in fact tend to lower the counts). 
Some proposed solutions are that the thick disk scale height varies with radius, or that the outer halo is lumpy. Either may well be possible. For example, fields at Galactic latitudes $|b|<25^{\circ}$ are now known to contain significant substructure towards the anticenter (Newberg et al. 2002; Ibata et al. 2003; Grillmair 2006a). More recently CADIS (Calar Alto Deep Imaging Survey; Phleps et al. 2005) mapped 1/30 2 in the magnitude range $15.5 \leq R \leq 23$, and found $n=-2.5$ if $q=0.6$.

\subsubsection{Panoramic surveys}

The advent of large galaxy surveys has been extraordinarily beneficial for Galactic astronomy. Although initially conceived to be a by product, multi-color deep wide field surveys have yielded completely new views of the Galactic halo. Some of the most spectacular results come from the Sloan Digital Sky Survey $\left(\mathrm{SDSS}^{2}\right)$. The first studies on the Early Data Release (EDR; $100^{\circ 2}$ down to $\left.r \sim 20\right)$ were carried out by Ivezić et al. (2000) using RR Lyrae; Yanny et al. (2000) using A-stars (BHBs and blue stragglers) and Chen et al. (2001) with stars of halo turn-off color. In agreement with previous work using the same technique (see previous section), Chen et al. (2001) measured $n=$ $-2.3 / 2.5$ and $q=0.6 / 0.5$ depending on the local normalization of the halo. However, Yanny et al. (2000) estimated $n=-3.2 \pm 0.3$ and $q=0.65$ (after masking out the overdensities due to the Sagittarius streams), in accordance with Ivezić et al. (2000) who found $n \sim-3$. Note that the latter two works employ tracers that probe considerably larger distances (particularly in comparison to the halo turn-off stars, which are intrinsically much fainter: $M_{V_{\mathrm{TO}}} \sim 4$ versus $M_{\mathrm{V}_{\mathrm{HB}}} \sim 0.6$; Binney and Merrifield 1998).

Using RR Lyrae stars, the QUEST survey (Vivas et al. 2004) find $n=-3.1 \pm 0.1$ (and that steeper profiles produce worse fits), and $q$ varying with radius as proposed by Preston et al. (1991).

The SDSS has now imaged $8,000^{\circ 2}$ of the sky yielding a truly panoramic view of the Galaxy (Adelman-McCarthy et al. 2007). However, instead of simplifying the picture and providing definitive answers, it has shown that the halo is far more complex than envisioned some ten years ago. For example, Newberg and Yanny (2006) find that there are significant asymmetries with respect to the line connecting the galactic center to the anticenter in the number counts of halo turnoff stars. They postulate this could be due to a triaxial halo. In concert with this, Xu et al. (2006) find an excess of stars towards $[l, b]=\left(270^{\circ}, 60^{\circ}\right)$ compared to $[l, b]=\left(90^{\circ}, 60^{\circ}\right)$.

Juric et al. (2008) have derived photometric parallaxes ${ }^{3}$ for 48 million stars in the SDSS catalog and attempted full modeling of the various Galactic components. These authors find that it is possible to measure the properties of the thin and thick disk relatively robustly (provided large portions of the celestial sphere have been observed). However they acknowledge that determining the properties of the halo is more difficult, partly because of the presence of significant clumps and overdensities, as shown in Fig. 2. The halo power law index $n$ is poorly constrained by the data, but an oblate halo with $q=0.5$ seems to be favored, although no good fits are obtained at the faint

\footnotetext{
2 http://www.sdss.org.

3 Assuming a "mean" photometric parallax relation, as function of color index, for all metallicities.
} 

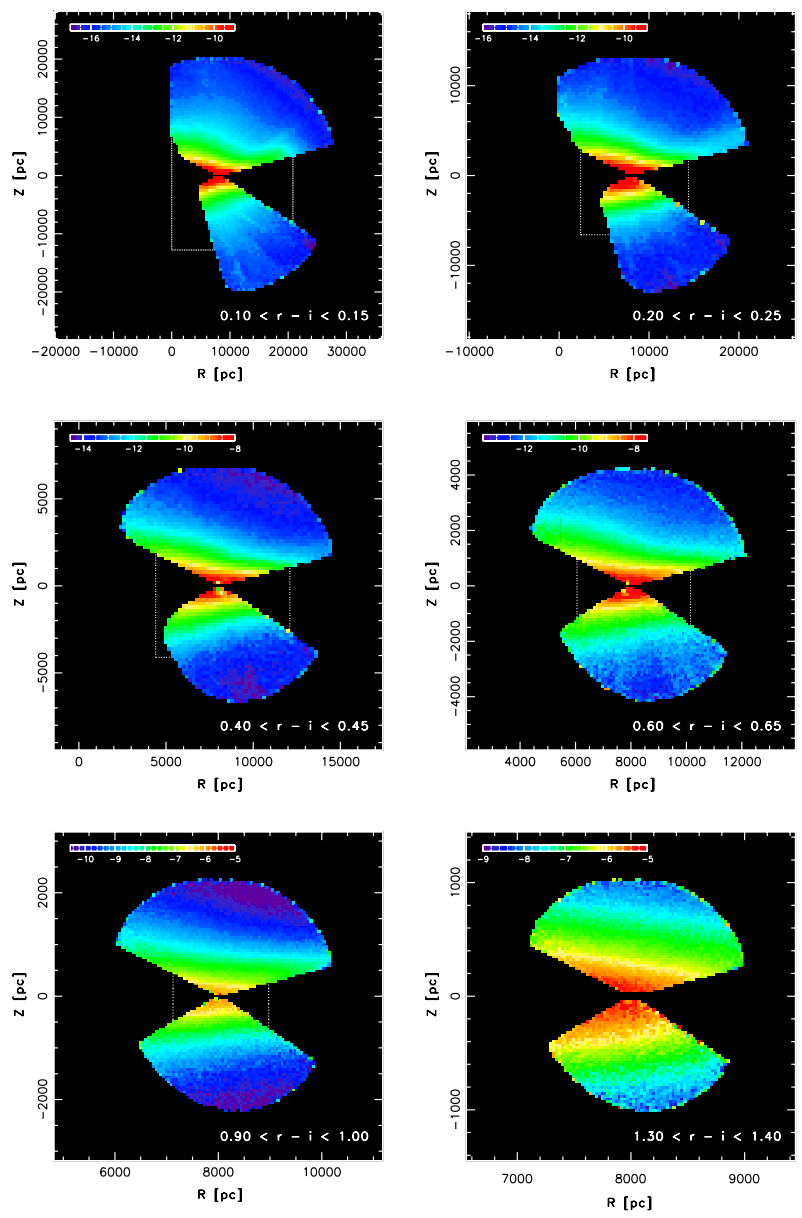

Fig. 2 Left panels: Stellar number density as a function of $(R, z)$ for different $r-i$ bins using SDSS photometric parallaxes (the distance scale greatly varies from panel to panel). Each white dotted rectangle denotes the bounding box of the region containing the data on the following panel. Note the large overdensities particularly in the top panels. Right panels: The stellar number density for the same color bin as in the top left panel $(0.10<r-i<0.15$, hence preferentially selecting stars near the halo turn-off point), shown here for cross-sections parallel to the Galactic plane, as a function of the distance from the plane (from $16 \mathrm{kpc}$ at the top left to $6 \mathrm{kpc}$ in the bottom right). The origin marks the Sun's position, while the circles aid in visualizing departures from axial symmetry of the Galaxy. Note the strong asymmetry with respect to the $y=0$ line, which is due to the Virgo overdensity. (From Juric et al. 2008. Courtesy of Mario Juric. Reproduced with permission of the AAS)

end. Amongst various structures, Juric et al. (2008) have detected a huge overdensity towards the constellation of Virgo at $l=270^{\circ}$ and $b=60^{\circ}$ (the same region that cannot be reproduced by smooth axisymmetric models).

The SDSS is focused on the northern galactic cap. This implies that the nature of the overdensities (global versus local) may be difficult to establish without good coverage of the southern hemisphere. In particular, if the halo were triaxial, one would 

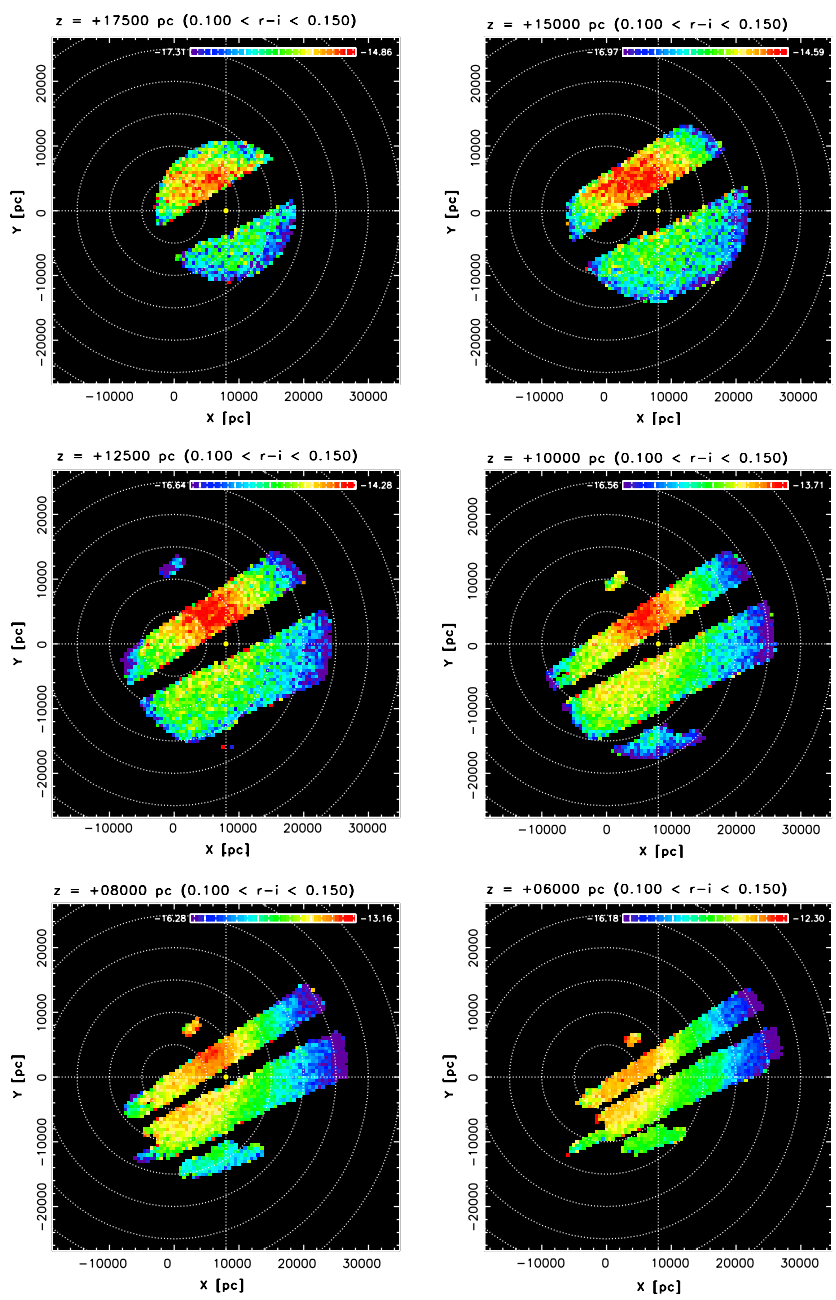

Fig. 2 continued

still expect (in the most reasonable case) symmetry with respect to the $z=0$ plane. $\mathrm{Xu}$ et al. (2007) carried out this exercise and compared star counts towards the North Galactic Pole (NGP) from SDSS and towards the South Galactic Pole (SGP) using SuperCOSMOS ${ }^{4}$ photographic data (Hambly et al. 2001). They found that there is an asymmetry between the north and southern cap of approximately $17 \%$ in the $B$-band. Therefore, they concluded that the Virgo overdensity is likely a foreign component of the stellar halo (see also Sect. 3). Their modeling, after subtracting the overdensities, favors an axisymmetric halo with parameters $n=-2.8$ and $q=0.7$.

This last example shows particularly well the need for large sky coverage and deep photometry to establish the structure of the stellar halo of the Galaxy. This is the only

$\overline{4 \text { http://www-wfau.roe.ac.uk/sss/. }}$ 
way to break the known degeneracies affecting the modeling of number counts from restricted pencil beam surveys. Nevertheless, given the problems in reproducing star counts at the faint end and the discovery of large overdensities on the sky, it is essential that the presence of substructure is taken into account in the models.

At this point in time, it transpires that the inner halo may well be axisymmetric and flattened ( $q \sim 0.6)$, while the outer halo (traced by e.g. RR Lyrae stars and very deep counts as in Robin et al. 2007) may be more spherical (and steeper). It would be interesting to explore whether this is due to different formation paths for the inner and outer halo (e.g. dissipative versus non-dissipative formation, see Sect. 6.1). The gravitational pull of the stellar disk (and the adiabatic contraction of the halo) may also be (partly) responsible for this dichotomy, see e.g. Binney and May (1986); Chiba and Beers (2001). In principle, it is also possible that the dual shape of the stellar halo reflects a change in the number of progenitors and their orbital characteristics with distance from the Galactic center.

\subsection{Normalization}

The local normalization (density of the stellar halo at the position of the Sun) has been determined both locally as well as from deep pencil beam surveys. Morrison (1993) isolated local halo giants from an objective-prism survey of metal weak stars, and derived a halo number density expressed in terms of halo-to-disk ratio of 1:1,200 for $\mathrm{K}$ giants $\left(M_{V} \leq 0.5\right)$, and 1:850 for dwarf stars with magnitudes $5 \leq M_{V} \leq 8$. The latter is comparable to the estimate obtained by Fuchs and Jahreiß (1998) using nearby M dwarfs (within $25 \mathrm{pc}$ ), who measured $\rho_{0}=1.5 \times 10^{-4} \mathrm{M}_{\odot} / \mathrm{pc}^{3}$ (see also Digby et al. 2003). On the other hand, Flynn et al. (1996) used very deep HST counts of M dwarfs and obtained a factor four smaller number of red dwarfs, in agreement with Gould et al. (1998) who find $\rho_{0} \sim 6.4 \times 10^{-5} \mathrm{M}_{\odot} / \mathrm{pc}^{3}$ using subdwarfs in 53 fields observed with the Wide Field Planetary Camera on the Hubble Space Telescope (HST). Attempts to measure the normalization using BHB stars are more tricky because of the variation of the properties of the horizontal branch with age and metallicity. This implies that the transformation from number of BHBs to $M_{\odot}$ is non-trivial. Note that the measurement of $\rho_{0}$ relies on an accurate determination of the luminosity function (and hence on the slope of the initial mass function), but possibly the largest source of discrepancy is the varying shape of the halo with radius. For example, the deep counts using HST explore larger distances, and hence presumably also the rounder part of the stellar halo, and therefore necessarily favor a rather low local normalization (Fuchs and Jahreiß 1998).

\subsection{Kinematics}

Obtaining space velocities for halo stars is no doubt more laborious than mapping their sky distribution. Although radial velocities are relatively straightforward to obtain for a small number of objects even for rather faint stars (e.g. $V \sim 19$ take less than 1 hourlong integrations with $4 \mathrm{~m}-8 \mathrm{~m}$ class telescopes), measuring their tangential velocities is only possible if both the proper motion and distance are known. While estimates of the latter may be obtained through photometric or spectroscopic parallaxes, proper 
motions from the ground require a large time baseline (of the order of 100 years) to produce milliarcsecond accuracy (see, e.g. Beers et al. 2000 and references therein). Therefore, and for many other reasons (Lindegren 2005), astrometric space missions with wide field angle capabilities are an absolute necessity.

Presently the catalogs of halo stars with relatively accurate 3D kinematics are somewhat small, and typically sample a few kpc around the Sun. They have been constructed using proper motions mainly from Hipparcos, and radial velocities from other surveys. Velocity errors are in the range $20-30 \mathrm{~km} \mathrm{~s}^{-1}$. Currently the largest such catalog available is that by Beers et al. (2000), with approximately one thousand nearby stars with $[\mathrm{Fe} / \mathrm{H}]<-0.6$ dex.

\subsubsection{Local halo}

Local samples of halo stars show a small amount of prograde rotation $V_{\phi} \sim 30-50$ $\mathrm{km} \mathrm{s}^{-1}$ (Carney et al. 1996; Layden et al. 1996; Chiba and Beers 2000; Morrison et al. 2008a). The velocity ellipsoid is roughly aligned with a cylindrical coordinate system, having dispersions of $\left(\sigma_{R}, \sigma_{\phi}, \sigma_{z}\right)=(141 \pm 11,106 \pm 9,94 \pm 8) \mathrm{km} \mathrm{s}^{-1}$ (Chiba and Beers 2000). The stellar halo is therefore supported by random motions, and its flattened shape is consistent with the degree of velocity anisotropy observed (see Sect. 2.3.3, also Sommer-Larsen and Christensen 1989).

The velocity distribution of halo stars near the Sun appears to be fairly smooth, with only a small degree of substructure (see Sect. 3 for more details). There is no strong correlation of the rotational velocity $V_{\phi}$ with metallicity. However, at large distances above the Galactic plane ( $\sim 3-5 \mathrm{kpc})$, the halo becomes slightly retrograde (Majewski et al. 1996; Carney et al. 1996, see also Kinman et al. 2007), and its mean metallicity is lower (Carollo et al. 2007; Morrison et al. 2008a).

\subsubsection{Global properties}

Figure 3 shows the line of sight velocities of a sample of 240 objects in the Galactic halo (Battaglia et al. 2005, 2006) as function of distance from the Galactic center. This sample includes K giant stars from the Spaghetti survey, field horizontal branch stars, globular clusters and dwarf galaxies. On the right-hand side panel, the radial velocity dispersion is plotted. This quantity shows an almost constant value of $120 \mathrm{~km} \mathrm{~s}^{-1}$ out to $30 \mathrm{kpc}$ and then continuously declines down to $50 \mathrm{~km} \mathrm{~s}^{-1}$ at about $120 \mathrm{kpc}$ (see Sommer-Larsen et al. 1997 for an earlier similar conclusion from a smaller and less distant sample). This fall-off puts important constraints on the density profile and total mass of the dark matter halo of the Milky Way. In particular, under the assumption of a power-law density for the stellar halo and a constant velocity anisotropy, an isothermal profile can be ruled out, while both a dark halo following a truncated flat model (Wilkinson and Evans 1999) of mass $5_{-1.7}^{+2.5} \times 10^{11} \mathrm{M}_{\odot}$ and an NFW profile of mass $9.4_{-0.9}^{+1.4} \times 10^{11} \mathrm{M}_{\odot}$ and concentration $c=18$ are consistent with the data (see also Moore et al. 2006; Abadi et al. 2006).

The stellar halo is not completely smooth kinematically (see Sect. 3). Evidence of halo moving groups was presented by Ratnatunga and Freeman (1985) towards the 
Fig. 3 Radial velocities in the Galactic Standard of Rest $V_{\mathrm{GSR}}$ as function of distance from the Galactic center (top); and Galactocentric radial velocity dispersion of the Milky Way stellar halo (bottom). The curves show the best-fit models obtained assuming a truncated flat dark matter halo of mass $5_{-1.7}^{+2.5} \times 10^{11} \mathrm{M}_{\odot}($ solid curve $)$ and an NFW profile of mass $9.4_{-0.9}^{+1.4} \times 10^{11} \mathrm{M}_{\odot}$ and $c=18$ (dotted curve), with constant anisotropy. The strong decline suggests that for an NFW profile to provide a better fit either the anisotropy must change with radius, or the stellar halo must have an edge. (From Battaglia et al. 2005, 2006. Courtesy of Giuseppina Battaglia. Reproduced with permission of Wiley-Blackwell Publishing)
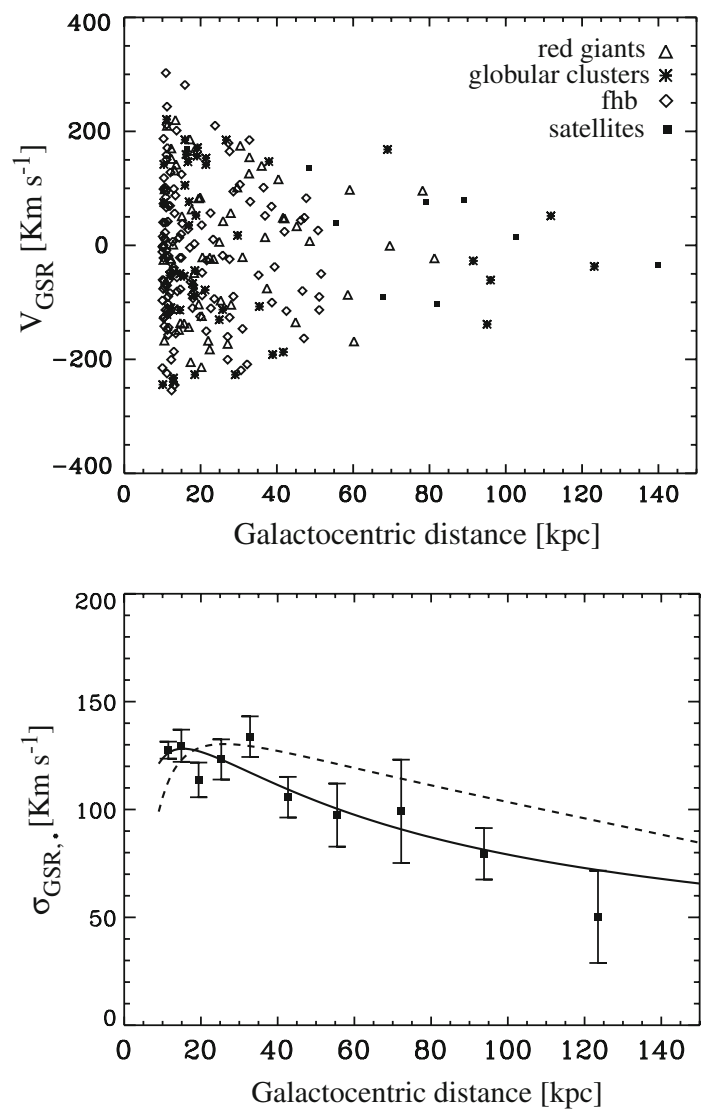

South Galactic Pole using radial velocities for K giants, by Majewski et al. (1996) towards the North Galactic Pole based on proper motions, and more recently by e.g. Clewley and Kinman (2006), Sirko et al. (2004) and Vivas et al. (2004) using horizontal branch stars' radial velocities. We discuss these substructures in more detail in Sect. 3 .

\subsubsection{A small digression on the relation between the shape of the stellar halo and its velocity ellipsoid in the Solar neighborhood}

As described above, the stellar halo does not show significant rotation. This implies that its flattened shape has to be attributed to anisotropy in the velocity ellipsoid. ${ }^{5}$

We will now derive the expected relation between the flattening of the stellar halo and the degree of anisotropy using the virial theorem. In steady state, the virial theorem

\footnotetext{
5 A similar argument is used to explain the shape of elliptical galaxies, see Binney and Tremaine (1987).
} 
in tensor form may be expressed as

$$
2 T_{j k}+\Pi_{j k}+W_{j k}=0
$$

where

$$
\begin{aligned}
T_{j k} & =\frac{1}{2} \int \mathrm{d}^{3} x \rho \bar{v}_{j} \bar{v}_{k} \\
\Pi_{j k} & =\int \mathrm{d}^{3} x \rho \sigma_{j k}^{2} \\
W_{j k} & =\int \mathrm{d}^{3} x \rho x_{k} \frac{\partial \Phi}{\partial x_{j}}
\end{aligned}
$$

with $\rho(\mathbf{x})$ the density profile of the system, $\bar{v}_{i}$ the mean velocity along the $i$-axis, $\sigma_{j k}^{2}$ its velocity tensor, and $\Phi(\mathbf{x})$ the gravitational potential of the dominant mass component. Note that in the case of the stellar halo, the density and the gravitational potential are not related through Poisson's equation; the stellar halo is a tracer population embedded in an underlying potential whose main contributor is the dark matter halo.

If we assume that the dark halo is spherically symmetric and that the stellar halo is axisymmetric, then $W_{x x}=W_{y y}$ and $W_{j k}=0$ for $j \neq k$. Similar arguments can be used to show that all equations are automatically satisfied for $j \neq k$, while the only two independent equations that remain are

$$
\begin{aligned}
2 T_{x x}+\Pi_{x x}+W_{x x} & =0 \\
2 T_{z z}+\Pi_{z z}+W_{z z} & =0 .
\end{aligned}
$$

Because the streaming (mean) motion of the stellar halo is negligible compared to the random motions, we may set $T_{x x}=T_{z z}=0$. In this case,

$$
\frac{\Pi_{x x}}{\Pi_{z z}}=\frac{W_{x x}}{W_{z z}}
$$

which states that shape of the mass-weighted velocity dispersion tensor depends on the shape of the (moments of the) mass-weighted gravitational potential. Equation (3) is equivalent to

$$
\frac{\int \mathrm{d}^{3} x \rho(R, z) \sigma_{x}^{2}}{\int \mathrm{d}^{3} x \rho(R, z) \sigma_{z}^{2}}=\frac{\int \mathrm{d}^{3} x \rho(R, z) \frac{x^{2}}{r} \frac{\mathrm{d} \Phi}{\mathrm{d} r}}{\int \mathrm{d}^{3} x \rho(R, z) \frac{z^{2}}{r} \frac{\mathrm{d} \Phi}{\mathrm{d} r}} .
$$

Note that $\sigma_{x} \neq \sigma_{z}$ if the density distribution of the tracer is axisymmetric. For simplicity we will assume

1. The shape of the velocity ellipsoid does not depend on location

$$
\sigma_{x}^{2} / \sigma_{z}^{2}=\text { constant }
$$


2. A logarithmic potential for the dark halo

$$
\Phi(r)=v_{c}^{2} \ln \left(r^{2}+d^{2}\right)
$$

3. The density profile of the stellar halo follows a power-law, is axisymmetric and has flattening $q$. Using Eq. (1) we obtain

$$
\rho(R, z)=\rho_{0}\left(m / m_{0}\right)^{n}, \quad m^{2}=R^{2}+z^{2} / q^{2} .
$$

Under these conditions, the left-handside of Eq. (4) reduces to a constant. The goal now is to evaluate the right-handside expression and compare it to the numerical value derived observationally for this constant.

Let us consider the case in which $n=-4$. This value is somewhat steeper than that discussed in Sect. 2.1, but it has the advantage of allowing the integrals to be solved analytically, and hence leads to a simpler expression. In this case Eq. (4) becomes

$$
\frac{\sigma_{x}^{2}}{\sigma_{z}^{2}}=\frac{1}{4 q^{2}\left(1-q^{2}\right) \operatorname{arctg}\left[\sqrt{\frac{1-q}{1+q}}\right]}\left\{q \sqrt{1-q^{2}}+2\left(1-2 q^{2}\right) \operatorname{arctg}\left[\sqrt{\frac{1-q}{1+q}}\right]\right\}
$$

The observed flattening of $q \sim 0.6$ therefore translates into a predicted $\sigma_{x}^{2} / \sigma_{z}^{2}=$ $1 / 2\left(\sigma_{x}^{2}+\sigma_{y}^{2}\right) / \sigma_{z}^{2}=1 / 2\left(\sigma_{R}^{2}+\sigma_{\phi}^{2}\right) / \sigma_{z}^{2}=1.73$. The measured value of $1 / 2\left(\sigma_{R}^{2}+\right.$ $\left.\sigma_{\phi}^{2}\right) / \sigma_{z}^{2}=1.76 \pm 0.37$ (Chiba and Beers 2000) is interestingly close. This presumably also means that the effective Galactic potential near the Sun is quite close to that of an isothermal sphere (see also White 1985; Sommer-Larsen and Christensen 1989).

\section{Substructure}

One of the most fundamental ingredients of the $\Lambda \mathrm{CDM}$ cosmological model is that galaxies grow via mergers. Therefore, perhaps the most direct way of testing this paradigm is by quantifying the amount of mergers that galaxies have experienced over their lifetime. This implies finding the traces of those merger events.

Mergers are expected to have left large amounts of debris in the present-day components of galaxies. In particular, a stellar halo is easily built up via the superposition of disrupted satellites (Johnston et al. 1996; Helmi and White 1999; Bullock et al. 2001; Harding et al. 2001). This is clearly exemplified in Fig. 4 adapted from the simulations and semi-analytic modeling by Bullock and Johnston (2005).

The prediction that debris in the halo should be ubiquitous in hierarchical cosmologies, together with the discoveries of a disrupting dwarf galaxy in the halo by Ibata et al. (1994) as well as of nearby remains from an ancient accretion event by Helmi et al. (1999), have boosted the search for substructure in this component of our Galaxy in recent years. Such searches have benefited enormously from the advent of large 
Fig. 4 A "simulated" stellar halo built up from accreted satellites. The color scale indicates surface brightness. (Sanjib Sharma, Kathryn Johnston and James Bullock are acknowledged for this figure)

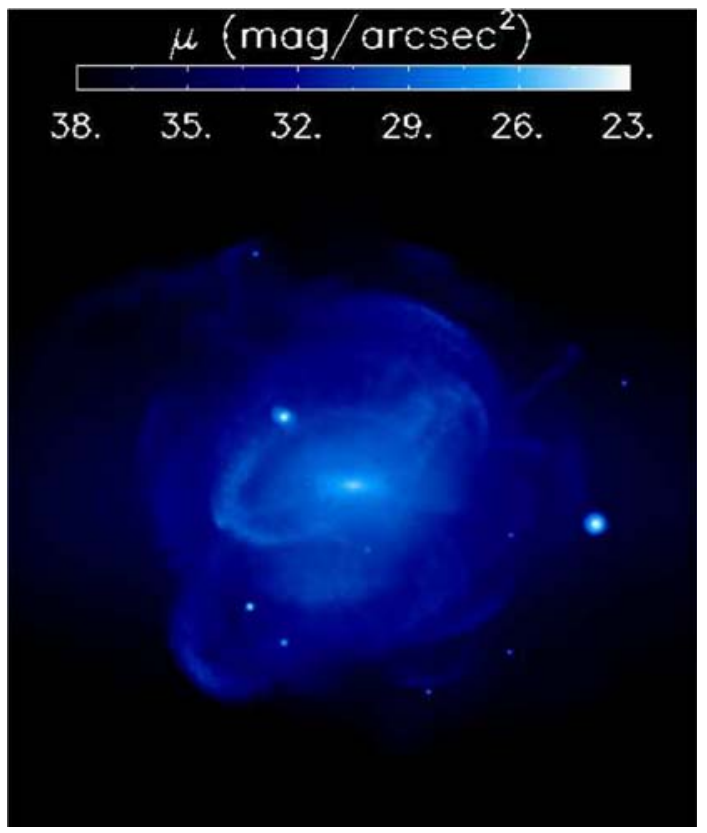

photometric surveys, and the SDSS in particular, as we shall see below, have all led to a shift in paradigm in the field over the past 10 years.

From a theoretical point of view, one expects substructures in the spatial domain, as well as in velocity space (or in phase-space). One can distinguish essentially two regimes in which substructures will be most easily found in either of these domains:

$-t \sim t_{\text {orb }}$ Short after infall, or for satellites orbiting the outer halo (dynamical timescales are comparable to the time since infall). In this case, streams are very coherent in space. Hence, a simple way to reveal these accretion events is to map of the positions of halo stars on the sky (Johnston et al. 1996).

$-t>>t_{\text {orb }}$ Long after infall, or in the inner halo $(r<15-20 \mathrm{kpc})$. The debris is spatially well-mixed, as shown in Fig. 5. Because of the conservation of phasespace density, the stars in a stream will have very similar velocities (see bottom panels in Fig. 5). In fact, streams get colder as time goes by. Therefore the characteristic signature of an accreted galaxy, even many Gyr after infall, is the presence of very cold structures in velocity space (Helmi and White 1999).

\subsection{In the spatial domain}

Because of the relative ease with which young substructures may be uncovered in the spatial domain, the largest fraction of known streams are those that are spatially coherent.

These discoveries did not really happen systematically until the advent of deep photometric wide-field surveys. The Sagittarius (Sgr) dwarf galaxy was a true beacon, 

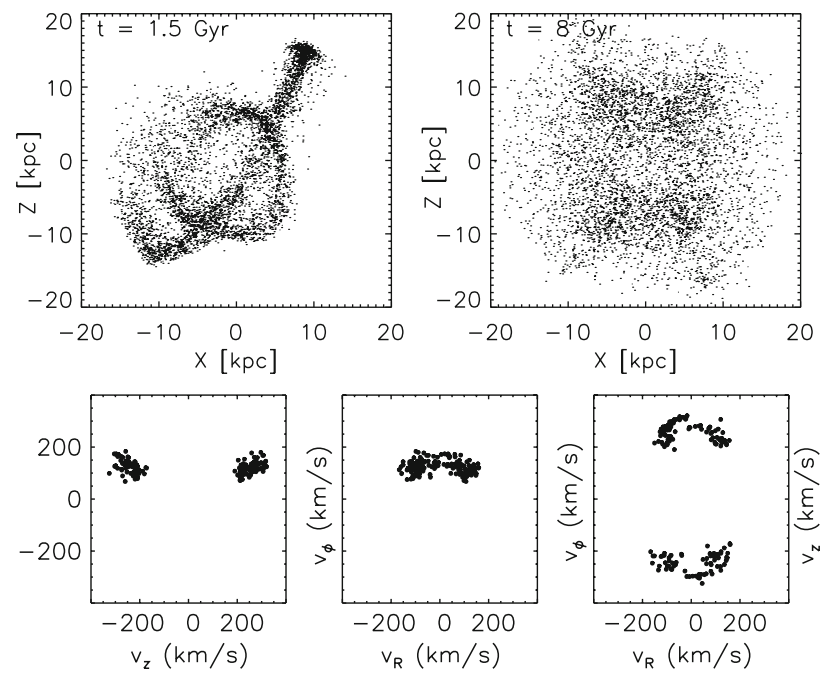

Fig. 5 Simulation of the disruption of a satellite galaxy orbiting in the inner regions of the halo of the Milky Way. The top panels show the $x z$ projection of the particles' positions, which evidence significant evolution over short timescales. The velocities of the particles located in a spherical volume of $\sim 2 \mathrm{kpc}$ around the Sun at $t \sim 8 \mathrm{Gyr}$ are strongly clustered, as shown in the bottom panels. Based on the simulation from Helmi et al. (1999)

because it was clearly tidally disturbed (Ibata et al. 1994; Gómez-Flechoso et al. 1999). Shortly after its discovery, a southern extension was found by Mateo et al. (1998). Somewhat later, Majewski (1999) reported debris at $40^{\circ}$ further south from the main body, which could be tentatively fit by models of a disrupting dwarf (Johnston et al. 1999). A very large excess of RR Lyrae and BHB stars at high Galactic latitudes was subsequently discovered in the SDSS survey (Ivezić et al. 2000; Yanny et al. 2000). This overdensity was roughly along the north-south elongation of the Sgr dSph, but now at $60^{\circ}$ away from it, and at a significantly larger distance $(50 \mathrm{kpc}$ instead of $15 \mathrm{kpc}$ from the Galactic center). The association of the overdensity to the Sgr dwarf galaxy northern/leading stream was nearly obvious (see e.g. Gómez-Flechoso et al. 1999), as the models of Helmi and White (2001) showed. ${ }^{6}$

After this, the Spaghetti Survey also found an overdensity at the same location using red giant branch stars with measured radial velocities (Dohm-Palmer et al. 2001). Martínez-Delgado et al. (2001) using deep color-magnitude diagrams detected the presence of a main sequence and turn-off at similar locations, and Ibata et al. (2001) using carbon stars were able to trace the stream over an extended portion of the sky.

A spectacular view of the Sgr streams was given by the Two Micron All Sky Survey (2MASS). ${ }^{7}$ Majewski et al. (2003) using M giants (selected from the 2 MASS

\footnotetext{
6 This was posted as a preprint on astro-ph in March 2000; just as the SDSS discoveries were being made.

7 http://pegasus.phast.umass.edu/.
} 


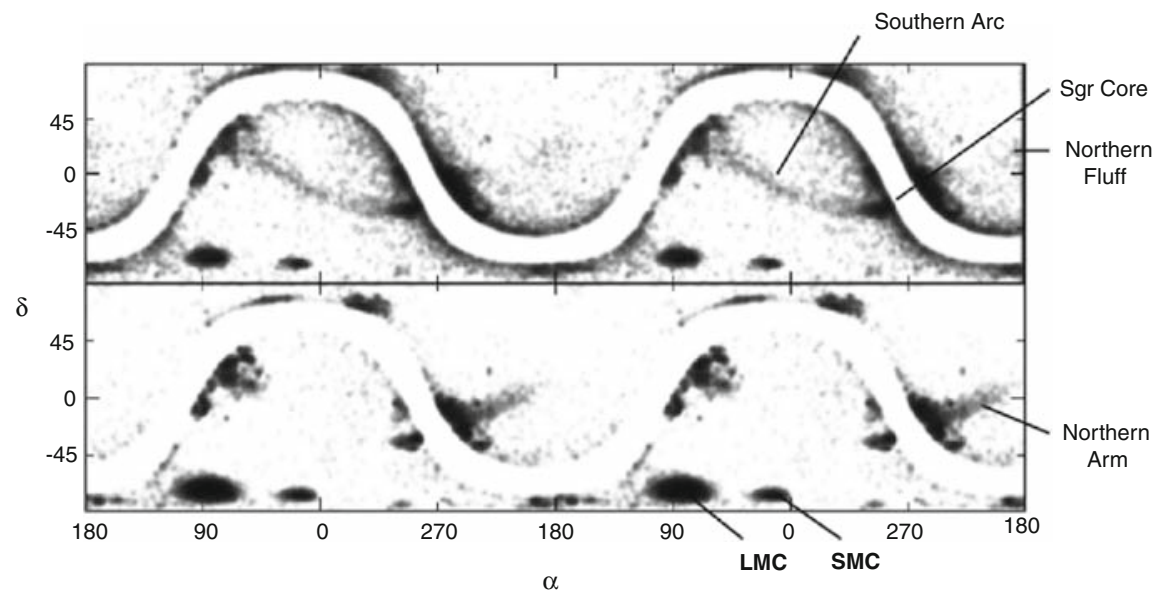

Fig. 6 2MASS revealing the streams from Sgr. Smoothed maps of the sky for point sources selected according to $11 \leq K_{S} \leq 12$ and $1.00<J-K_{S}<1.05$ (top), and $12 \leq K_{S} \leq 13$ and $1.05<J-K_{S}<1.15$ (bottom). Two cycles around the sky are shown to demonstrate the continuity of features. (From Majewski et al. 2003. Courtesy of Steve Majewski. Reproduced with permission of the AAS)

database on the basis of their colors) were able to map the streams $360^{\circ}$ around the sky as shown in Fig. 6. The vast extent of the Sgr dwarf streams has been used to constrain the shape of the Galactic gravitational potential, albeit with conflicting results (Helmi 2004b; Johnston et al. 2005; Fellhauer et al. 2006).

In the years to follow, many of the structures discovered in the halo would be linked to the Sgr debris (Newberg et al. 2002; Vivas et al. 2001; Martínez-Delgado et al. 2004). An exception to this is the Monoceros ring (Yanny et al. 2003; Ibata et al. 2003), a low-latitude stream towards the anticenter of the Galaxy, that spans about $100^{\circ}$ in longitude at a nearly constant distance. It is somewhat of a semantic distinction to state that this structure is part of the stellar halo, or of the Galactic thin or thick disks. As yet, the nature of the feature and of the Canis Major overdensity in a similar location at low Galactic latitude (Martin et al. 2004), are highly debated. Possible interpretations are that it is debris from an accreted satellite (e.g. Helmi et al. 2003; Martin et al. 2004; Peñarrubia et al. 2005; Martínez-Delgado et al. 2005), an overdensity associated to the Galactic warp (Momany et al. 2004, 2006), or even a projection effect caused by looking along the nearby Norma-Cygnus spiral arm (Moitinho et al. 2006). Recently, Grillmair (2006a) has mapped the structure of the Monoceros overdensity using SDSS, and showed that it consists, at least in part, of a set of very narrow low-latitude substructures (see Fig. 7), which can easily be explained by the models of Peñarrubia et al. (2005). It is worthwhile mentioning here that such a satellite may well perturb the Galactic disk, and induce departures from axial symmetry or overdensities such as e.g. Canis Major. Furthermore, the Sgr dwarf leading tail also appears to be crossing the Galactic plane at roughly the same location and distance as the ring (Newberg et al. 2007), and may well be responsible for some of the substructures observed. 


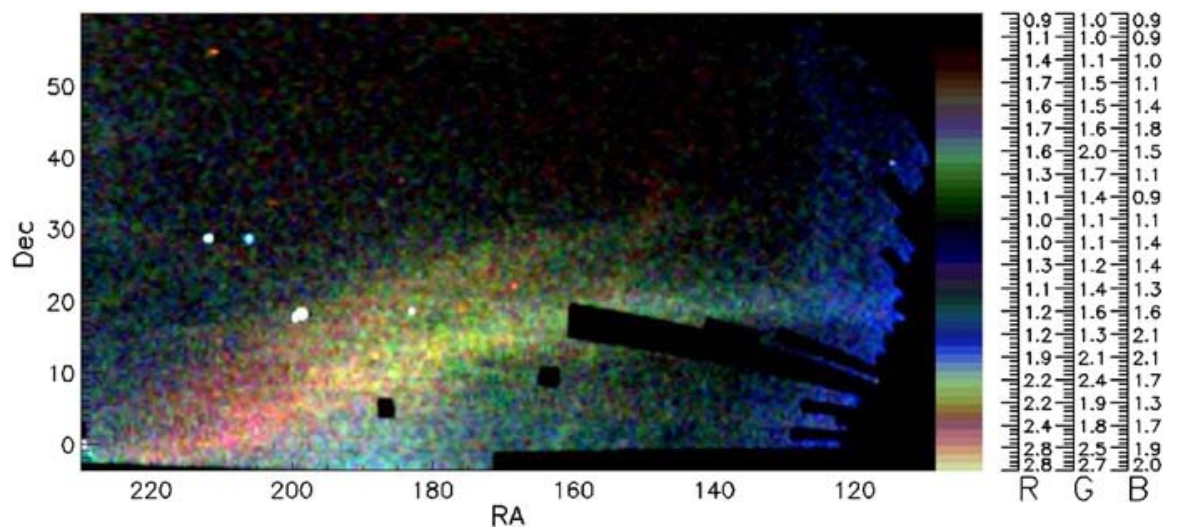

Fig. 7 The field of streams from SDSS featuring Sgr, several new satellites and the low-latitude Monoceros ring. (From Belokurov et al. 2006b. Courtesy of Vasily Belokurov. Reproduced with permission of Wiley-Blackwell Publishing)

At the time of writing of this review, other substantial substructures have been uncovered, such as the Virgo overdensity (Juric et al. 2008), possibly linked to the Virgo Stellar Structure (Vivas et al. 2001; confirmed by Duffau et al. 2006 using radial velocities), the Triangulum-Andromeda (Rocha-Pinto et al. 2004) and the Hercules-Aquila clouds (Belokurov et al. 2007b). The nature of these substructures is unclear, particularly because of the large area on the sky that they cover. It is evident that detailed kinematic studies of large samples of stars with good distances, as well as chemical abundances, and full coverage of the structures, are necessary to understand whether these are truly related to tidal debris. In this case, one would expect to see coherent motions, as indeed appears to be the case for the Hercules-Aquila cloud.

Narrow streams have been detected around globular clusters (Grillmair et al. 1995), the most spectacular examples being Pal 5 (Odenkirchen et al. 2001) and NGC5466 (Grillmair and Johnson 2006; Belokurov et al. 2006a). This shows that globular clusters probably also have contributed to the halo field population (Baumgardt et al. 2008). Another example of a narrow tidal tail is the Orphan-Stream (Grillmair 2006a; Belokurov et al. 2007c), whose progenitor may well have been a dwarf spheroidal-like object accreted by the Galaxy several Gyr ago (Sales et al. 2008).

It is very important to understand what kind of biases affect our ability to detect substructure, particularly if our goal is to determine the mass function of the objects accreted and the timing of these events. For example, it is obviously easier to detect high surface brightness features like those originating in massive recently accreted satellites. For instance, the Sagittarius stream reaches a surface brightness of $\Sigma_{V} \sim 28 \mathrm{mag} / \mathrm{arc}-$ $\sec ^{2}$ at $l \sim 350^{\circ}$ and $b \sim 50^{\circ}$ (Martínez-Delgado et al. 2001), where it was first detected, while the other overdensities discovered so far typically have surface brightnesses $\Sigma_{V} \sim 32-33 \mathrm{mag} / \mathrm{arcsec}^{2}$ (see Fig. 8 in Belokurov et al. 2007a and private communication). 


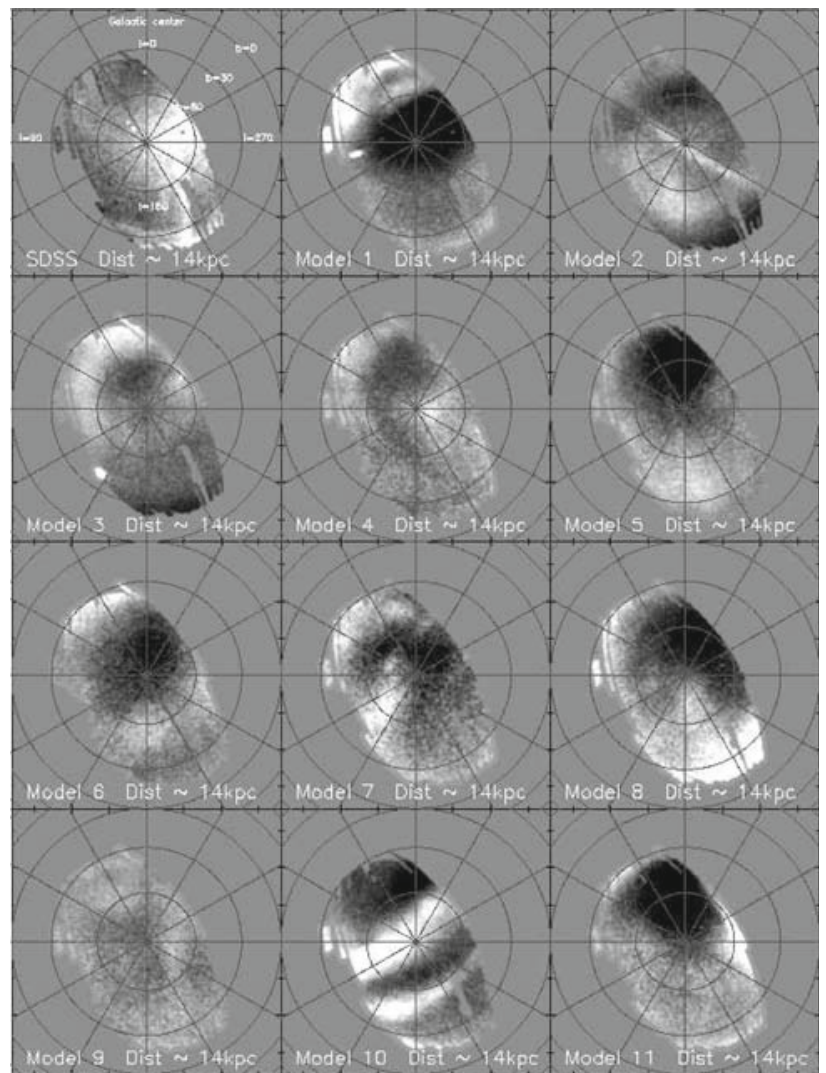

Fig. 8 Residuals (SDSS or simulations minus the smooth model) smoothed using a $\sigma=42^{\prime}$ Gaussian from the best oblate model fits for the SDSS data (top left panel) and for the 11 simulations from Bullock and Johnston (2005). Only the $20 \leq r<20.5$ slice, corresponding to heliocentric distances $\sim 14 \mathrm{kpc}$ are shown. (From Bell et al. (2007). Courtesy of Eric Bell)

The large majority of the above-mentioned substructures are located in the outer halo, at distances $\gtrsim 15 \mathrm{kpc}$ from the Galactic center. The inner halo, on the other hand, appears to be relatively smooth (Lemon et al. 2004).

\subsection{In the velocity domain}

As briefly discussed in Sect. 2.3, kinematic information is more expensive to obtain, and hence the velocity domain has been less explored despite its significantly larger suitability for searches of substructure.

Two new major spectroscopic surveys are likely to change this in the nearby future. The Sloan Extension for Galactic Understanding and Exploration (SEGUE, ${ }^{8}$

\footnotetext{
8 http://www.sdss.org/segue/aboutsegue.html.
} 
Beers et al. 2006) and the RAdial Velocity Experiment (RAVE, ${ }^{9}$ Steinmetz et al. 2006) are both currently collecting spectra for very considerable samples of stars. The final catalogs will be highly complementary. SEGUE will observe $\sim 240,000$ stars in the range $15<V<21$, while RAVE aims at $10^{6}$ stars with $9<I<12$. The average velocity errors that these surveys can achieve are of the order of 10 and $1 \mathrm{~km} \mathrm{~s}^{-1}$, respectively. The advent of such large spectroscopic surveys is clearly a very interesting development since the kinematics of halo stars can be used to find and constrain the nature of the overdensities, as well as to measure the mass distribution in our Galaxy (e.g. Smith et al. 2007).

The catalogs of halo stars with 3D kinematics used so far in searches of substructure are significantly smaller and less accurate (see for example, the work of Chiba and Yoshii 1998; Helmi et al. 1999; Chiba and Beers 2000; Kepley et al. 2007). Thanks to the developments mentioned above, however, we may expect soon a ten-fold increase in the number of nearby halo stars with full kinematics. For example it will be possible to combine the RAVE radial velocities with the relatively accurate proper motions from the Tycho-2 catalog. (Carollo et al. 2007 have already demonstrated the potential of combining the SDSS data (with radial velocities and stellar parameters) with proper motions from the USNO-B catalog, to understand the global structure of the halo, see Sect. 4.2.1. Note that this dataset is not optimal to search for substructure in the Solar neighborhood because of the relatively large velocity errors, which are typically in the range $30-50 \mathrm{~km} \mathrm{~s}^{-1}$ ).

So far, there have only been a few detections of substructure near the Sun. This is not really surprising. Because of the short dynamical timescales in the inner Galaxy (a typical halo star near the Sun will have made between 50 and 100 revolutions in a Hubble time), each accreted satellite will generally give rise to multiple streams (or wraps of debris) that will cross the Solar neighborhood, as shown in Fig. 5. In a stellar halo completely built from disrupted galaxies, the predicted total number of nearby streams is

$$
N_{\text {streams }} \sim\{300-500\}(t / 10 \mathrm{Gyr})^{3}
$$

i.e., a few hundred streams are expected to cross the solar neighborhood after a Hubble time (Helmi and White 1999). This quantity is only weakly dependent on the number and mass spectrum of the accreted satellites. Furthermore, in the context of the $\Lambda \mathrm{CDM}$ model, it is worthwhile stressing that these streams are expected to originate in only a handful of objects (Helmi et al. 2003).

To detect these streams, samples with approximately 5,000 halo stars are needed (that is, $N_{\text {sample }} \sim 10 \times N_{\text {streams }}$ ). Given the size of the samples currently available, we could clearly be in the limit in which essentially each stream is populated by just one star.

Nevertheless, Helmi et al. (1999) successfully detected two streams coming from a common satellite accreted 6-9 Gyr ago (Kepley et al. 2007). A simulation to reproduce its properties is shown in Fig. 5. The reason that these authors were able to detect the streams is three-fold. Firstly, they studied the distribution of stars in an "integrals

\footnotetext{
9 http://www.rave-survey.aip.de/rave/.
} 
of motion" space (defined by two components of the angular momentum), where stars from a common progenitor are very strongly clustered (Helmi and de Zeeuw 2000). Thus they could map the two streams, one with 4 stars and one with 8 stars, to one overdensity with 12 stars. The second reason for the successful detection is that the stream stars are going through their pericenters, and at the orbital turning points one expects an enhancement in the density of a stream. (This phenomenon is in essence the same as the shells observed around elliptical galaxies, Quinn 1984). This explains why the streams detected by Helmi et al. (1999) were not really that cold. The third reason is that the values of the integrals of motion of the accreted stars are quite different from those typical of solar neighborhood halo stars-lying in the tails of the distribution, exactly where one expects the most recently accreted objects to be.

Seabroke et al. (2008) have recently put constraints on the presence of nearby massive halo streams using the radial velocities of stars towards the Galactic poles. They find no vertical flows with densities greater than $1.4 \mathrm{stars} / \mathrm{kpc}^{3}$. This rules out the possibility that streams from the Sagittarius dwarf are crossing the Solar neighborhood, which in turn implies that models in which the dark matter halo is prolate are favored (Helmi 2004b; Law et al. 2005).

Seabroke's results are in excellent agreement with the arguments of Gould (2003), who constrained the degree of granularity using the revised New Luyten Two-Tenths (rNLTT) catalog of proper motions of 4,500 nearby halo stars. He finds, using statistical arguments, that no streams crossing the Sun contain more than 5\% of the nearby halo stars. This is also in unison with the results of Helmi et al. (1999).

As mentioned above, further detections of substructure will require significantly larger samples with full and accurate 3D kinematics. The first steps towards this will come with RAVE and SEGUE, but the clear breakthrough will be made by the European Space Agency's mission Gaia (Perryman et al. 2001). Gaia is scheduled for launch in 2011 and will provide high accuracy astrometry (at the 10 microarcsecond level for $V \leq 12$ stars, and 200 microarcseconds for $V \sim 20$ ) and multi-color photometry for over 1 billion stars throughout the Milky Way. Radial velocities at 10 $\mathrm{km} \mathrm{s}^{-1}$ accuracy will be acquired for stars down to $V \sim 16-17$. Therefore, the Gaia dataset will allow us to quantify the amount of substructure in the halo of our Galaxy, and consequently its evolutionary path.

\subsection{Implications}

Much of the work in the field of "substructure" has so far been rather qualitative in nature. The discoveries of the substructures are clearly the first step, but what do they actually tell us about how our Galaxy formed? This requires a more quantitative approach.

Bell et al. (2007) have used SDSS data to compute the rms fluctuations in the number of objects compared to a smooth halo model. They find typical values of $\sim 40 \%$. They interpret this using the simulations of Bullock and Johnston (2005), where this statistic can vary from 20 to $80 \%$ depending on the detailed halo formation history. This could imply that the (outer) Galactic halo is fairly average in its history and could have been built completely by accreted satellites. However, a careful comparison of the overall 
structure of the fluctuations shows that there may still be room for a smooth halo component, as illustrated in Fig. 8. For example, there are significant under-densities in the Bullock and Johnston (2005) maps, which are not apparent to the same extent in the SDSS data.

Starkenburg et al. (2008) use a different approach and data from the Spaghetti Survey to quantify the amount of substructure. Their sample contains approximately 100 red giant stars with radial velocity and distance information. They develop a statistic called the 4-distance, which is essentially a correlation function in a 4D space:

$$
4 d_{i j}=\sqrt{a_{\omega}\left(\omega_{i j}\right)^{2}+a_{d}\left(d_{i}-d_{j}\right)^{2}+a_{v}\left(v_{\mathrm{rad}, i}-v_{\mathrm{rad}, j}\right)^{2}}
$$

where $\omega_{i j}$ denotes the angular separation on the sky of star $i$ with respect to star $j, d_{i}$ its distance and $v_{\mathrm{rad}, i}$ its radial (line-of-sight) velocity. The coefficients $a_{x}$ denote weights to normalize the various observables and to account for the observational errors. Substructure would manifest itself in an excess of nearby pairs in this 4D space. Using this method, Starkenburg et al. (2008) find that $\sim 20 \%$ of the stars are in substructures, the most prominent of which corresponds to the Sgr stream northern overdensity (Dohm-Palmer et al. 2001). When comparing to the simulations of halos built up completely from accreted satellites of $10^{7} \mathrm{M}_{\odot}$ by Harding et al. (2001), Starkenburg et al. (2008) find that these typically show a significantly larger amount of substructure. Only in about $10 \%$ of the cases, the characteristics of such halos are similar to what is measured by the Spaghetti survey. They interpret this as meaning that if the whole halo was built from accreted satellites, these must have left behind relatively broad streams, implying that either they were relatively large or have orbited the Galaxy long enough to be significantly broadened. The other possible interpretation is that there is an underlying smooth component in the halo. Starkenburg et al. (2008) were clearly limited by the small sample size, which provided significant Poisson noise to their statistical estimations. This situation is likely to change in the very near future with SEGUE, which will provide orders of magnitude larger samples of red giant stars with accurate distances and velocities (Morrison et al. 2008b).

\section{Ages and chemical abundances of halo stars}

\subsection{Age distribution}

The determination of the age of individual stars is one of the most difficult measurements in astrophysics. Some possible techniques include isochrone fitting of individual stars (their absolute magnitude, effective temperature and metallicity needs to be accurately known) or star clusters; asteroseismology and nucleochronology.

Direct age measurements can be inferred by comparing the observed abundance ratios of the radioactive elements of e.g. ${ }^{232} \mathrm{Th}$ (half-life of $14 \mathrm{Gyr}$ ) and ${ }^{238} \mathrm{U}(4.5 \mathrm{Gyr}$ ) with theoretical predictions of their initial production. The first measurements of very metal-poor nearby stars have yielded ages of 15.2 \pm 3.7 Gyr (Sneden et al. 1996), $14 \pm 3$ Gyr (Cayrel et al. 2001; Hill et al. 2002), and 13.2 Gyr (Frebel et al. 2007). 
The white dwarf luminosity function, and especially its cut-off, also provides strong constraints on the age of the population to which they belong (see, e.g. Chaboyer 1998). This method, based on the white dwarf cooling sequence, has been used in the past to derive the age of the oldest stars in the Galactic disk (see e.g. Hansen and Liebert 2003). However, the scarcity of known halo white dwarfs and the lack of good kinematical data to distinguish these from the far more common disk stars have so far, prevented such a measurement for the halo (Torres et al. 1998).

The horizontal branch morphology of a stellar population at a given abundance tends to be bluer for older populations, and redder for younger ones (Layden 1995). In the halo, there is some indication that the horizontal branch morphology of field stars becomes redder with Galactocentric distance. If this is interpreted as due to age, it means that the outer halo would be younger by a few Gyr, compared to the inner halo (see e.g. Searle and Zinn 1978; Suntzeff et al. 1991). It should be noted, however, that the existence of an age spread or a gradient in the population of halo globular clusters is still under debate (e.g. Sarajedini et al. 1997; Stetson et al. 1996; De Angeli et al. 2005).

\subsection{Chemical abundances}

\subsubsection{Metallicity distribution}

The local halo metallicity distribution peaks at a value $[\mathrm{Fe} / \mathrm{H}] \sim-1.6 \mathrm{dex}$, and extends well below $[\mathrm{Fe} / \mathrm{H}] \sim-3$ dex (e.g. Ryan and Norris 1991).

Recently Carollo et al. (2007) based on SDSS data revived the idea that the halo may be described by two broadly overlapping components. The inner halo peaks at $[\mathrm{Fe} / \mathrm{H}] \sim-1.6 \mathrm{dex}$, is flattened and slightly prograde, while the outer halo would peak around $[\mathrm{Fe} / \mathrm{H}] \sim-2.2 \mathrm{dex}$, would be rounder and in net retrograde rotation. Although a dichotomy in the Galactic halo had been suggested before (see also Sect. 2.1), this has now been confirmed with a much larger sample of stars covering a large area on the sky and is now clearly reflected also in the metallicity distribution as shown in Fig. 9.

Because of the importance of metal-poor stars in constraining the formation history of the Galaxy, as well as the physics of the high-redshift Universe (e.g. reionization, initial mass function, etc.) significant efforts have been made to better characterize the metallicity distribution at the very metal-poor end. More recently, the HK and the Hamburg/ESO (HES) surveys have yielded a dramatic increase in the number of nearby metal-poor stars known (Beers and Christlieb 2005). In these surveys, metal-poor candidates are selected from objective-prism spectra for which the $\mathrm{Ca}, \mathrm{H}$ and $\mathrm{K}$ lines are weaker than expected at a given $(B-V)$ color. These stars are then followed up with medium-resolution spectroscopy (see, for example, Frebel et al. 2006).

These surveys have demonstrated two important points. First, three extremely metal-poor stars with $[\mathrm{Fe} / \mathrm{H}]<-4.75$ dex have been discovered, all of which are carbon rich. Secondly, no metal-free stars (Population III) have been found thus far.

These results could in principle, put strong constraints on the initial mass function (IMF). For example, Salvadori et al. (2007) find, using semi-analytic models of galaxy formation, that the lack of metal-free stars constrains the critical metallicity for 

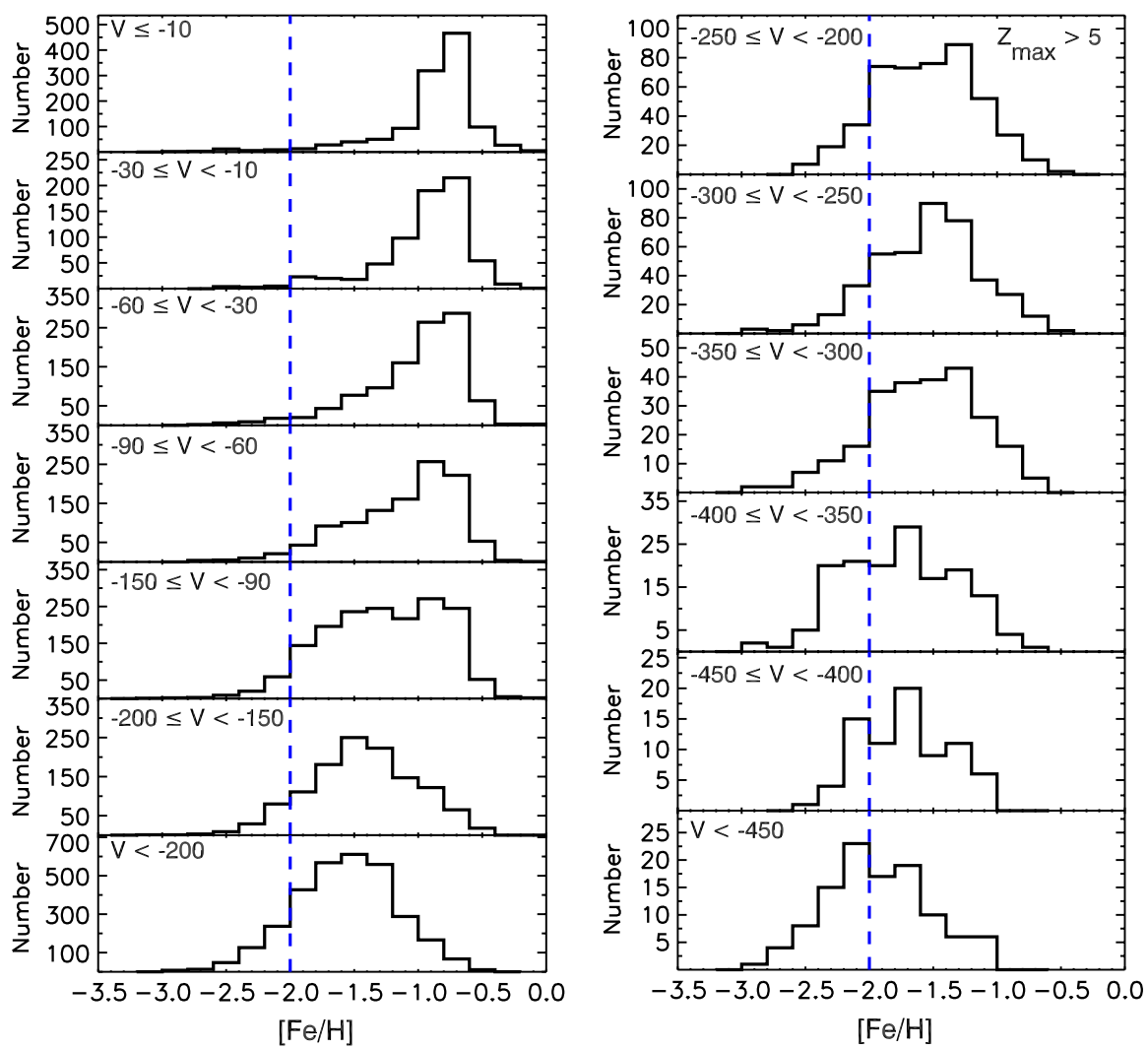

Fig. 9 Metallicity distribution function based on medium-resolution spectroscopy for $2 \times 10^{4}$ nearby stars obtained with SDSS. The histograms show that the inner halo $\left(V \sim-200 \mathrm{~km} \mathrm{~s}^{-1}\right)$ exhibits a peak metallicity $[\mathrm{Fe} / \mathrm{H}]=-1.6$, and that the outer halo (right panels) peak at a value $[\mathrm{Fe} / \mathrm{H}]=-2.2$, and have a net retrograde rotation. (From Carollo et al. 2007. Courtesy of Daniela Carollo. Reproduced with permission of Nature)

low-mass star formation to be $Z_{\mathrm{cr}}>0$ (see Schneider et al. 2002), or the masses of the first stars to be $M_{\text {PopIII }}>0.9 \mathrm{M}_{\odot}$. Brook et al. (2007) reach a similar conclusion using cosmological hydrodynamical simulations.

From a theoretical point of view, the initial mass function of Population III stars is expected to be top-heavy, with masses in the range $100-1000 \mathrm{M}_{\odot}$, e.g. Bromm et al. (1999); Abel et al. (2000). Such stars are predicted to leave very characteristic imprints in the abundance patterns of the next generation of stars (a very strong oddeven effect, e.g. Heger and Woosley (2002)). However, such patterns are not consistent with those observed for example by Cayrel et al. (2004) for nearby very metal-poor stars. Furthermore, the observed trends can be explained by normal supernovae with masses $<100 \mathrm{M}_{\odot}$ (Umeda and Nomoto 2005; Silk and Langer 2006).

The solution to this conundrum is still to be found. Given the small number statistics, the IMF appears to be consistent with Salpeter as well as with a top-heavy functional form (Salvadori et al. 2007). On the other hand, the high incidence of carbon-enhanced, 
unevolved stars among extremely metal-poor stars has been used to propose that the IMF in the early Galaxy was shifted toward higher masses (Lucatello et al. 2005). Based on the fact that the two most metal-poor stars known are both in binary systems (and that their high $[\mathrm{C} / \mathrm{Fe}]$ could be due to the winds from an AGB companion star), Tumlinson (2007) discusses that an IMF with a characteristic mass $\sim 3 \mathrm{M}_{\odot}$ would reproduce the properties and distribution of the halo stars at the metal-poor end.

In the coming years, our knowledge of the halo metallicity distribution function is likely to improve significantly with surveys such as Skymapper, ${ }^{10}$ SEGUE and LAMOST, ${ }^{11}$ which will extend by large factors the currently known handful of extremely metal-poor stars (Christlieb 2006). These new datasets will give us to access to the very high-redshift Universe through the fossil record left by the first generations of stars ever formed.

\subsubsection{Elemental abundances}

Much more information about the chemical history of the Galactic halo may be obtained from detailed studies of the elemental abundances patterns. This is because the various chemical elements are synthesized by different processes in stars of different masses and on different timescales (McWilliam 1997). For example, $\alpha$-elements $(\mathrm{O}, \mathrm{Mg}, \mathrm{Si}, \mathrm{Ca}, \mathrm{S}$, and $\mathrm{Ti})$ are produced during the explosion of a massive star as a Supernova Type II, which occurs only a few million years after its formation. Ironpeak elements, on the other hand, are produced both by Type Ia and Type II supernovae. Type Ia supernovae are the result of a thermonuclear explosion induced by the transfer of mass onto the surface of a white dwarf by a binary companion star. This implies that these explosions take place typically on a longer timescale, of the order of 0.1 to a few Gyr (e.g. Matteucci and Recchi 2001). Heavier elements beyond the iron-peak are created by neutron capture, through slow (s) and rapid (r) processes. The s-process can take place when the neutron flux is relatively low, i.e. the timescale between neutron captures is large compared to that of the beta decay. These conditions are found in the envelopes of Asymptotic Giant Branch (AGB) stars, and are most efficient in stars with masses $3-5 \mathrm{M}_{\odot}$, implying a timescale of $100 \mathrm{Myr}$ after the stars were born (see Goriely and Siess 2005 and references therein). On the other hand, the r-process occurs when the neutron flux is sufficiently high to allow for rapid neutron captures. This is believed to occur in environments like those produced by supernovae Type II. It should be stressed, however, that the exact sites and conditions under which the various neutron-capture elements are produced, particularly at very low metallicities, are still somewhat uncertain (Travaglio et al. 2004; Arnould et al. 2007).

Figure 10 shows how the chemical enrichment depicted by the $[\alpha / \mathrm{Fe}]$ versus $[\mathrm{Fe} / \mathrm{H}]$ may proceed in a given object depending on its initial mass function (which sets the initial level of $[\alpha / \mathrm{Fe}]$ ) and its star formation rate (which sets at what metallicity the "knee" is observed).

\footnotetext{
10 http://www.mso.anu.edu.au/skymapper/.

11 http://www.lamost.org.
} 


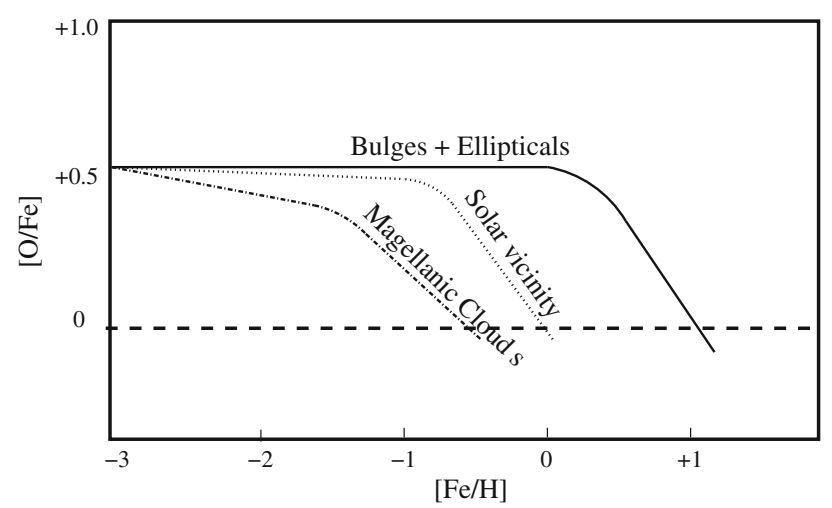

Fig. 10 Sketch of the predicted $[\mathrm{O} / \mathrm{Fe}]$ versus $[\mathrm{Fe} / \mathrm{H}]$ relation in different systems as a consequence of their different enrichment timescales. The $\alpha$ enhancement seen in the Galactic bulge can be explained by an intense star formation burst in which mainly supernovae Type II polluted the interstellar medium. On the other hand, the Magellanic Clouds had a lower star formation rate, and so most of their stars have a relatively enhanced iron-content. Thin-disk stars near the Sun are an intermediate case. (Based on Matteucci 2001)

Interestingly, the majority of the local halo stars are $\alpha$-enhanced (Wheeler et al. 1989; Nissen et al. 1994; Carretta et al. 2000), where typically $[\alpha / \mathrm{Fe}] \sim 0.3$ dex. This implies that the formation timescale for these stars was very short, less than $1 \mathrm{Gyr}$. This argument is based on the fact that the $\alpha$-enhancement may also be seen as an iron deficiency. Such a configuration may be obtained when the interstellar medium (ISM) has been enriched by supernovae Type II, but not significantly by Type Ia (Gilmore and Wyse 1998).

More detailed insights have been provided by Cayrel et al. (2004) and François et al. (2007). They have obtained high-resolution spectra $(R \sim 45,000)$ for 30 very metalpoor giants $(-4.1<[\mathrm{Fe} / \mathrm{H}]<-2.7 \mathrm{dex})$. Cayrel et al. (2004) find that the scatter in several $[\alpha / \mathrm{Fe}]$ and $[\mathrm{Fe}-$ peak/Fe] ratios is consistent with being only due to observational errors, as shown in Fig. 11 (see also Cohen et al. 2004). This would suggest that these stars formed in an extremely well-mixed medium, i.e. the cosmic scatter expected from the pollution by supernovae of different masses is not observed. In contrast, the neutron-capture elements behave very differently, showing a large spread at low metallicity (Barklem et al. 2005; François et al. 2007 and references therein). It is currently unclear how to reconcile these results. In a scenario in which the halo was formed via the accretion of several independent objects, one would naively expect that each of these objects followed their own chemical evolution path, hence a superposition of their debris should give rise to a large scatter in the various chemical abundance patterns. Perhaps the observed large scatter in [neutron-capture/Fe] would be consistent with such a scenario (see Cescutti et al. 2006), but why is there not a spread in other elements? Uncertainties in how nucleosynthesis proceeds at very low metallicities, as well as in modeling the chemical evolution in the proto-galaxy in a cosmological context, are likely to be both elements of this puzzle.

The chemistry of stars can also be used to trace the merging history of the Galactic halo (Freeman and Bland-Hawthorn 2002). For example, Nissen and Schuster (1997) found a correlation for local halo stars such that those with large apocentric distances 
Fig. 11 Elements ratios as function of $[\mathrm{Fe} / \mathrm{H}]$ for the sample of very metal-poor stars from Cayrel et al. (2004). Ca is an $\alpha$ element, while $\mathrm{Cr}$ is from the iron-peak, yet in both cases the scatter is extremely small. In contrast, neutron-capture element ratios such as [Y/Fe] and $[\mathrm{Ba} / \mathrm{Fe}]$ show very large scatter in the same stars (François et al. 2007). (From Cayrel et al. 2004. Courtesy of Rogier Cayrel. Reproduced with permission of A\&A)
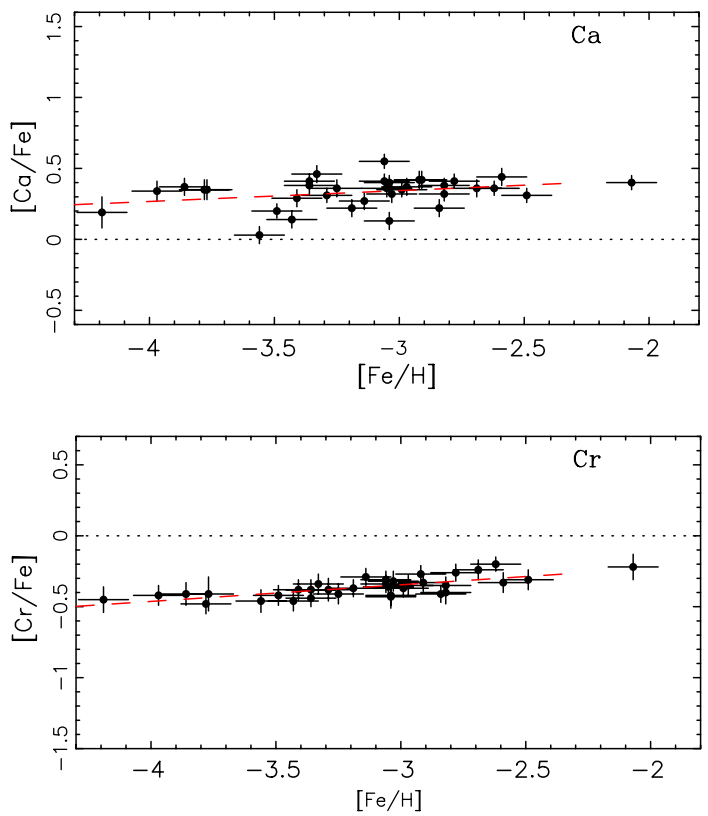

have relatively low $[\alpha / \mathrm{Fe}]$. This may be an indication that these anomalous halo stars have been accreted from dwarf galaxies with a different chemical evolution history (see also Fulbright 2002; Ivans et al. 2003). This is consistent with the fact that stars in dwarf spheroidal galaxies, tend to have lower $[\alpha / \mathrm{Fe}]$ at a given metallicity compared to the halo. This in turn has been used to show that dwarf galaxies could not have been significant contributors of the stellar halo if accreted after they formed a significant fraction of their stars (Shetrone et al. 2001; Tolstoy et al. 2003; Venn et al. 2004).

More recent abundance studies of large samples of stars in several dwarf spheroidals have demonstrated that these objects have similar initial levels of $[\alpha / \mathrm{Fe}]$ at very low [Fe/H], as shown in Fig. 12 (Tolstoy et al. 2006; Letarte 2007; Hill et al. 2008). It is likely that their relatively low star-formation rates have led to slow chemical enrichment, implying low values of $[\alpha / \mathrm{Fe}]$ compared to the halo for all but the oldest (or most metal-poor) stars in the dwarf spheroidals (Tolstoy et al. 2003).

\section{The satellites: dwarf galaxies and globular clusters}

Approximately 150 globular clusters and about 20 satellite galaxies are found in the Galactic halo. It is interesting to study their distribution as well as their internal properties to elucidate the evolutionary path of the Galaxy. In particular, it is worthwhile comparing these objects' stellar populations to the halo field stars, since in the most naive interpretation of the hierarchical paradigm, the stars have their origin in small galaxies, presumably akin to a certain (to be defined) extent the dwarf satellites we see around us today. 


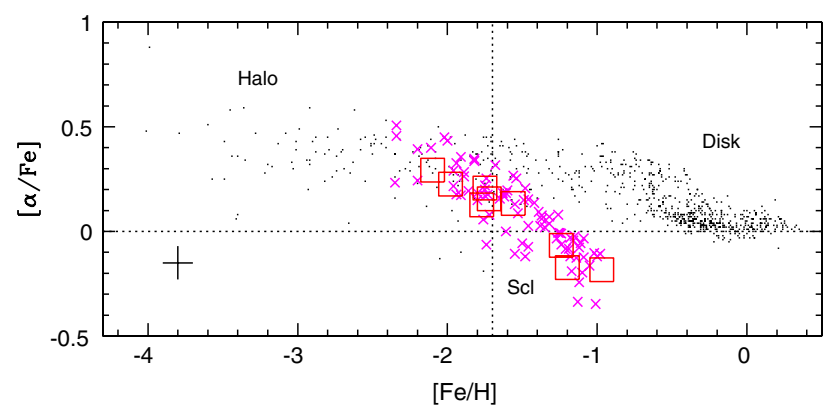

Fig. $12 \alpha$-abundance (average of $\mathrm{Ca}, \mathrm{Mg}$ and $\mathrm{Ti}$ ) as function of $[\mathrm{Fe} / \mathrm{H}]$ for 92 member stars in a central field of Sculptor (crosses, from Hill et al. 2008) compared to those in our Galaxy (dots; from the compilation of Venn et al. 2004). The open squares are previous measurements of individual stars (Shetrone et al. 2003). The most metal-poor stars in the dwarf spheroidals are as $\alpha$ enhanced as field stars in the Galactic halo. It is only at higher $[\mathrm{Fe} / \mathrm{H}]$ that the $[\alpha / \mathrm{Fe}]$ is significantly lower in the dwarf spheroidals (Tolstoy et al. 2006). (Courtesy of Eline Tolstoy and Vanessa Hill)

The number of known dwarf galaxies in the Local Group has doubled in the past few years, particularly at the faint luminosity end, thanks to the discovery of a large number of very low surface brightness satellites (e.g. Belokurov et al. 2007a; Martin et al. 2007). Despite this recent large increase in the abundance of Galactic satellites, this is still well-below the number of bound substructures orbiting galaxy-size halos in pure cold dark matter simulations (Koposov et al. 2007). This discrepancy has been termed the "missing satellites problem" (Klypin et al. 1999; Moore et al. 1999). Several solutions have been put forward including, for example, a modification of the nature of dark matter (Spergel and Steinhardt 2000). On the other hand, the modeling of the baryonic physics at the low-mass end of the galaxy scale is far from complete, and this is where environmental effects and processes such as reionization, feedback from massive stars, etc. are likely to have a significant impact on the probability of a substructure to hold onto its gas and form stars (e.g. Mac Low and Ferrara 1999; Bullock et al. 2000; Kravtsov et al. 2004).

The newly discovered satellites have luminosities in between (although sometimes comparable to) globular clusters and the classic dwarf spheroidals, but they are significantly more extended than the former. They have extremely large mass-to-light ratios (Simon and Geha 2007), computed under the assumption of virial equilibrium (which may very well turn out to be a very poor approximation since these objects could well be tidal debris).

There are two very interesting properties of the spatial distribution of the satellites. Firstly, their distribution is rather centrally concentrated, in the sense that the median distance is less than 30-40\% of the virial radius (as computed by Battaglia et al. 2005, 2006). For comparison, satellite substructures around halos in dark matter cosmological simulations tend to be much more evenly distributed (Gao et al. 2004; Kang et al. 2005). This apparent discrepancy can be solved by assuming, for example, that the luminous satellites are embedded in those substructures that were most massive at the time they crossed the virial radius of the Galaxy. Also the radial distribution of the halo globular clusters follows very closely that of the field stars. This can be explained if 
these objects formed in a highly biased set of gas-rich proto-galaxies at high-redshift (Kravtsov and Gnedin 2005).

The second interesting property is that the satellites are quite anisotropically distributed (Hartwick 2000; Kroupa et al. 2005; Metz et al. 2007). This can be reconciled with cold dark matter models if these galaxies have fallen in from a few directions, perhaps in groups (Li and Helmi 2008), or along filaments (Libeskind et al. 2005; Zentner et al. 2005; Kang et al. 2005). It is unclear whether this peculiarity will remain once the sky coverage is more complete, and once a thorough survey of the faintest objects is available. Furthermore, establishing the reality of the dynamical families proposed by Lynden-Bell and Lynden-Bell (1995), is only possible with knowledge of the satellite's orbits. For this, precise tangential velocities (i.e. proper motions) are required. Unfortunately only a few satellite galaxies have proper motion measurements, mostly obtained using the Hubble Space Telescope (see e.g. Piatek et al. 2007 and references therein) and these are still affected by large uncertainties. However, in the coming decade, the situation is going to improve significantly thanks to Gaia.

In contrast, the dynamics of the (inner) halo globular cluster population is much better known. For example, Dinescu et al. (1999) has compiled a sample with $\geq 40$ clusters with well-measured tangential velocities. The measurements are relatively modern (i.e. made with high-precision measuring machines, such as used in the Southern Proper Motion survey), and are tied to an absolute reference frame using, e.g. galaxies and QSOs, or stars with known absolute proper motion from, e.g. the Lick Northern Proper Motion Program or Hipparcos (for more details, see e.g. Dinescu et al. 1999 and references therein).

The reliable measurements of the dynamics of globular clusters in combination with dedicated programs to characterize their properties, such as chemical abundance patterns, ages, horizontal-branch morphology, and structural parameters has prompted the search for correlations in the hope to trace Galactic history (e.g. Ashman and Zepf 1998; Mackey and Gilmore 2004 and references therein). This is much in the same spirit of the original work of Searle and Zinn (1978) and Zinn (1993).

A recent highlight is the discovery of multiple stellar populations in the most massive globular clusters (Lee et al. 1999). One proposed explanation (but not the only one, c.f. Baumgardt et al. 2008 and references therein) is that these might be remaining cores of disrupted nucleated dwarf galaxies, as is the case for M54 which is located in the Sagittarius dwarf itself (Layden and Sarajedini 2000). These peculiar globular clusters also show an extended horizontal branch (EHB), and the presence of double or broadened main sequences (Piotto et al. 2007). In fact, about $25 \%$ of the globular clusters in our Galaxy exhibit unusual EHBs, which has prompted the idea that they might have a unique origin. Furthermore, these globular clusters are on average more massive and share the random kinematics characteristic of normal globular clusters in the outer halo (Lee et al. 2007).

These results, as well as the evidence of dynamical relation between the Sagittarius dwarf and six Galactic globular clusters (Bellazzini et al. 2003), suggest an accretion origin for the outer halo clusters. This is also supported by the work of Mackey and Gilmore (2004), who conclude that all young halo clusters (with $[\mathrm{Fe} / \mathrm{H}]<-0.8 \mathrm{dex}$ and red $\mathrm{HB}$ morphology, more common in the outer halo) and $\sim 15 \%$ of the old halo clusters $([\mathrm{Fe} / \mathrm{H}]<-0.8$ dex and blue $\mathrm{HB}$ morphology) are of external origin. 


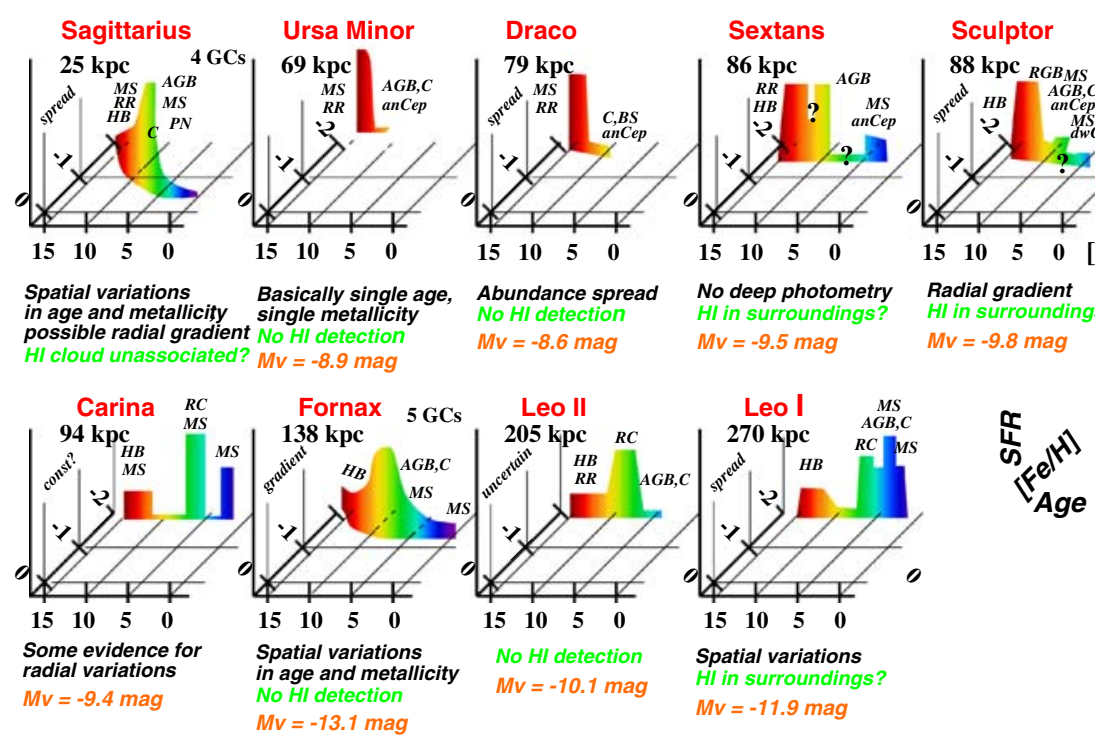

Fig. 13 Star formation histories of Milky Way dwarf spheroidal satellites. Each population box gives a schematic representation of star formation rate (SFR) as a function of age and metallicity. (From Grebel 2000. Courtesy of Eva Grebel, and Fabio Favata (editor))

Dwarf galaxies show, of course, much more complex stellar populations. Furthermore, these galaxies depict a large variety in star formation and chemical evolution histories (Grebel 2000; Dolphin et al. 2005), as shown in Fig. 13. Some dwarf galaxies appear to have stopped forming stars very early on, although it is clearly difficult to disentangle whether this happened at $z \sim 10$, i.e. before reionization, or at $z \sim 3-5$, i.e. after reionization (Gnedin and Kravtsov 2006). On the other hand, some dwarf galaxies have managed to form stars until very recently, such as Fornax (Stetson et al. 1998) or Sagittarius (Siegel et al. 2007).

There is, however, a remarkable uniformity in that all dwarf spheroidals studied so far show an absence of stars with metallicities below a value of $[\mathrm{Fe} / \mathrm{H}] \sim-3 \mathrm{dex}$ (Helmi et al. 2006). This could suggest a uniform pre-enrichment across the Local Group at high redshift, before the first generations of stars in these objects formed.

We are also interested in understanding the link between the field stars in the halo and those still bound to the satellites of the Milky Way (Sales et al. 2007). For example, the contribution of present-day dwarf galaxies to the halo near the Sun can be ruled out on the basis of the colors and chemical properties of the stellar populations (Unavane et al. 1996; Venn et al. 2004). These dwarfs have had a Hubble time to evolve in relative isolation, and it is reasonable that their stellar populations would not resemble those of objects disrupted a long time ago. However, one may wonder what the relation is between the progenitors of the dwarf galaxies and the halo building blocks. Under the assumption that the very metal poor stars represent the first generations of stars formed, the apparent lack of such objects in the dwarf spheroidals compared to the halo, implies that their progenitors were fundamentally different. One possible scenario is that the halo building blocks were associated to high-density peaks that 
collapsed at higher redshifts, while the dwarf galaxies presumably descend from more average density fluctuations in the early Universe (Helmi et al. 2006; Salvadori et al. 2008).

Nevertheless we know at least of one example of a significant contributor to the outer halo: the Sagittarius dwarf galaxy. Although it is currently unclear how massive this object was initially, current models postulate an initial stellar mass of $\sim 2-5 \times 10^{8} \mathrm{M}_{\odot}$ (Helmi 2004a; Law et al. 2005), which is slightly smaller but comparable to the total mass of the stellar halo obtained by extrapolating the local density and assuming a power-law distribution as described in Sect. 2. Therefore, given that we are just beginning to map the outer stellar halo in sufficient detail, it is very likely that much of this region of the Galaxy is the result of the accretion of dwarf galaxies like those we observe today.

\section{Formation scenarios}

There has been a tendency in the literature to classify formation scenarios according to two traditional models introduced in the 1960s and 1970s respectively, to describe the evolution of the Galaxy.

The first model was proposed by Eggen et al. (1962), where "the oldest stars were formed out of gas falling toward the galactic center in the radial direction and collapsing from the halo onto the plane. The collapse was very rapid and only a few times $10^{8}$ years were required for the gas to attain circular orbits in equilibrium". The influence of this work has been phenomenal, not just on models of the stellar halo, but also in the field of galaxy evolution in general. The second model, which is often used as a counter scenario, is that by Searle and Zinn (1978). On the basis of the lack of an abundance gradient for the outer halo globular clusters, these authors proposed that "these clusters formed in a number of small protogalaxies that subsequently merged to form the present Galactic halo". For many authors, this latter model is more reminiscent of the currently popular hierarchical paradigm. However, as we shall see below, ingredients of both models are likely to be present in the assembly of a Galactic system in a hierarchical cosmology.

It is therefore perhaps more instructive to distinguish models according to the degree of dissipation involved in the formation of a given component. In this context, the Eggen et al. (1962) model would fit in a category where the large majority of the nearby halo stars were formed during a dissipative process (namely the collapse of a gaseous cloud). On the other hand, in the Searle and Zinn (1978) model, the assembly of the outer halo would have proceeded without any dissipation.

\subsection{Global properties}

Several authors have attempted to model the formation and characteristics of the Galactic stellar halo. Different techniques have been applied, such as numerical simulations with and without gas physics and star formation, which are sometimes (fully) embedded in a cosmological context. Other attempts include the use of phenomenological descriptions of the evolution of baryons inside halos, usually in combination with an 
N-body simulation that provides the dynamical history of the system in the context of the hierarchical paradigm.

The global properties of the Galactic stellar halo, such as its shape, correlations with kinematics and metallicity distribution, have been reproduced in many of these works.

For example, Samland and Gerhard (2003) have modeled the formation of a disk galaxy in a slowly growing dark matter halo. In essence, this model is quite similar to the Eggen et al. (1962) scenario, but takes into consideration the fact that galaxies have grown in mass from the Big Bang until the present time. They follow the cooling of gas, star formation and feedback processes in great detail, and are able to obtain a realistic disk galaxy at the present time. In this simulation, the stellar halo defined by the stars with $[\mathrm{Fe} / \mathrm{H}]<-1.9$ dex is not rotating, while the more metal-rich stars with $-1.9<[\mathrm{Fe} / \mathrm{H}]<-0.85$ are rotating at $\sim 70 \mathrm{~km} \mathrm{~s}^{-1}$. The first component is slightly flattened in the same sense as the disk, while the second component is significantly flatter and largely supported by rotation. In this sense, one may argue that it resembles more closely a thick disk than the inner halo of the Milky Way.

More realistic attempts to simulate the formation of a disk galaxy like the Milky Way in a cosmological framework include those of Steinmetz and Muller (1995); Bekki and Chiba (2001) and Brook et al. (2003). These authors have modeled the collapse of an isolated, rigidly rotating sphere of $\sim 10^{12} \mathrm{M}_{\odot}$ onto which small-scale fluctuations according to a cold dark matter spectrum have been imposed. In these works the evolution of the dark matter and of the gas, star formation, etc. are followed self-consistently. In this sense they are, in principle, able to determine the relative importance of dissipative processes versus dissipationless mergers in the build-up of the halo. However, the numerical resolution in these studies is low (the spatial resolution, as measured by the softening, is typically $\sim 2.5 \mathrm{kpc}$ ), and the number of particles is small. For example, in Brook et al. (2003) the dominant disk component and the spheroid at the final time are represented by $\sim 4 \times 10^{4}$ star particles. This implies that the resolution is not sufficient to determine the detailed properties of the simulated galaxies robustly (e.g. small scale substructure such as dwarf galaxies and streams are not well resolved). Nevertheless, global (average) properties are likely to be reliable. For example, Steinmetz and Muller (1995) find in their simulations that the stellar halo (defined as the more metal-poor component) is triaxial in shape ( $c / a=0.65$ and $b / a=0.85$, with the minor axis roughly aligned with the angular momentum of the disk), and that the density profile is well-fit by a de Vaucouleurs law. In the simulation of Bekki and Chiba (2001) the outer halo is completely built up through accretion, while the inner halo results from merging between gas rich clumps. The inner halo is also adiabatically contracted by the formation of the disk (Binney and May 1986; Chiba and Beers 2001), which explains its more-flattened shape. In agreement with Steinmetz and Muller (1995), Bekki and Chiba (2001) find that the density profile varies from $r^{-3}$ at $r<10 \mathrm{kpc}$ to $r^{-4}$ at larger distances.

This is also in agreement with the simulations of Abadi et al. (2003). These authors have run fully cosmological hydrodynamical simulations of the formation of a disk galaxy. Although their final galaxies are of too early Hubble type (most of them have bulges that are too dominant compared to the disk component), they find some common (and hence presumably general) characteristics. The surface brightness profile 
of the outer halo components of these galaxies $(r \geq 20 \mathrm{kpc})$ can be fit with a Sersic law, with $\langle n\rangle=6.3$ and $\left\langle R_{\text {eff }}\right\rangle=7.7 \mathrm{kpc}$, i.e. $R^{-\overline{2} .3}$ at $\sim 20 \mathrm{kpc}$ and $R^{-3.5}$ around the virial radius. The shape of the outer halos is very mildly triaxial $\langle b / a\rangle=0.91$ and $\langle c / a\rangle=0.84$. These outer regions $(r \geq 20 \mathrm{kpc})$ contain mostly accreted stars (with fractions greater than $90 \%$ ), which originate in the most massive mergers that the central galaxy has experienced over its lifetime (the contribution of small accretion events may be underestimated because of resolution, but this is nevertheless unlikely to be the major channel by which galaxies grow). The inner halos are formed in-situ and are much more flattened with $c / a \sim 0.62$ (Abadi et al. 2006).

Diemand et al. (2005) provide an alternative view from dark matter only cosmological simulations. Following Moore (2001), they identify the most bound particles in halos that have collapsed at very high redshift (i.e. $2.5 \sigma$ peaks at $z=12$ ) and find that these end up in the inner regions of the most massive object at the present time. These particles (which are meant to represent stars) are distributed according to a very steep density profile $r^{n}$ with index $n \sim-3$ in analogy to the stars in the stellar halo of our Galaxy.

A complementary approach has been developed by Bullock and Johnston (2005) who follow the formation of the stellar halo purely built via accretion of satellites. The evolution of the baryonic components of the satellites (fraction of cold and hot gas, star formation and chemical enrichment histories) are followed through a phenomenological semi-analytic approach (as in White and Frenk 1991; Kauffmann et al. 1993; Somerville and Primack 1999). The satellites are placed on orbits (consistent with those found in cosmological simulations, e.g. Benson 2005), around a slowly growing gravitational potential that models the Galaxy (resembling the approach of Samland and Gerhard 2003). In this sense, they are limited to times after which the central potential has settled (i.e. after major mergers), and so, essentially they are well-placed to tackle the formation of the outer halo, i.e. $r>15-20 \mathrm{kpc}$. Through this modeling they find that the density profile of their stellar halos vary from $\rho \propto r^{-2}$ at $20 \mathrm{kpc}$ to $r^{-4}$ beyond $50 \mathrm{kpc}$.

The higher resolution and more detailed modeling of Bullock and Johnston (2005) also allows them to make predictions about the distribution of chemical abundances of the Milky Way outer halo stars. Font et al. (2006) find that the mean metallicity of the halo depends on the accretion history, and that gradients in metallicity of $\sim 0.5$ dex are not necessarily uncommon in their accreted halos. They predict that the stars in the outer halo should have lower $[\alpha / \mathrm{Fe}]$ than stars in the inner Galaxy. In their models, the most metal-poor stars are located in the inner $10 \mathrm{kpc}$, which at face value is opposite to the trend suggested by Carollo et al. (2007).

The hybrid cosmological approach of De Lucia and Helmi (2008) combines elements of the work of Diemand et al. (2005) and Bullock and Johnston (2005). These authors use a high-resolution cosmological simulation of the formation of a Milky Way-sized dark matter halo coupled to a semi-analytic galaxy formation code, to obtain insights into the global evolution of the Galaxy. By identifying the most bound particles of substructures in the simulation with the luminous cores of satellite galaxies, they are able to follow the structure but also the metallicity and age distribution of the "simulated" stellar halo. As shown in Fig. 14, also De Lucia and Helmi (2008) find that the stellar halo is much more centrally concentrated than the dark matter halo. 

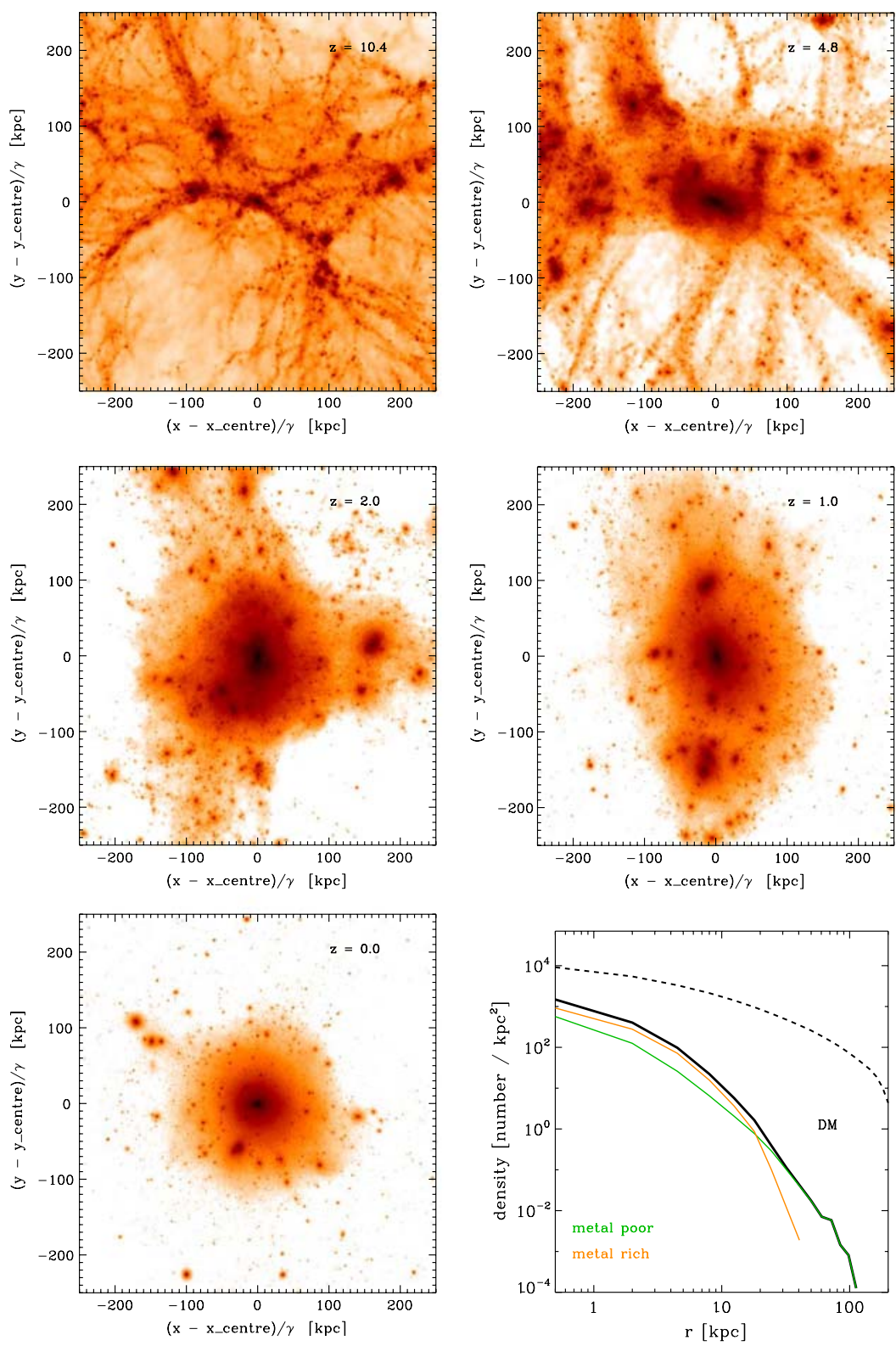

Fig. 14 Evolution of the dark matter distribution in a high-resolution cosmological simulation of the formation of a Milky Way-like halo by Stoehr et al. (2002). By identifying the cores of dark matter substructures with luminous satellites, De Lucia and Helmi (2008) can follow the formation and evolution of a spheroidal component, which would correspond to the stellar halo. For example, by analyzing where these objects deposit their debris, they can study the link between the chemistry and the structure of the stellar halo. This is shown in the bottom right panel, where the green and orange curves denote the surface density profile of metal-poor and metal-rich "stars", respectively. In comparison, the dashed black curve corresponds to the dark matter halo, which shows a significantly more shallow and extended distribution. (From De Lucia and Helmi 2008. Courtesy of Gabriella de Lucia) 
Furthermore, their inner halo is dominated by higher metallicity "stars", vis-a-vis the outer halo, with the transition occurring at $\sim 10-15 \mathrm{kpc}$ from the center, reminiscent of the findings of Carollo et al. (2007).

\subsection{Current status}

The discussion above shows that it is possible to reproduce the density profile and shape of the inner stellar halo in a model in which all stars were formed in situ as in Samland and Gerhard (2003), as well as in a model in which all stars were accreted as in Diemand et al. (2005), Bullock and Johnston (2005) and De Lucia and Helmi (2008). Furthermore, in all the models discussed here the oldest stars are located in the spheroid (halo + bulge), which implies that their age distribution and the broad chemical properties (low mean metallicity and $\alpha$-enhancement) are also likely to be reproduced. Nevertheless, much more work is required to understand the expected trends as well as predictions for the detailed chemical abundance patterns that have now been observed in such exquisite detail for nearby halo stars. The work of Chiappini et al. (1997) is an excellent example of how this can be done, but it needs to be embedded in a more cosmological framework.

The discussion in the previous paragraphs also shows that we do not really possess a clear view of what are the exact predictions of the hierarchical paradigm on the scales of the Milky Way and its satellites. The relatively simplistic and coarse fully cosmological simulations are limited in their predictive power at the level of detail that is required for the Milky Way galaxy. The semi-analytic approach of combining high-resolution dark matter simulations with phenomenological descriptions of the evolution of the baryonic components, is a powerful and promising technique, that will be developed much further in the near future (see e.g. De Lucia and Helmi 2008). The first attempts by Bullock and Johnston (2005) at modeling the outer halo have yielded extremely interesting results, despite the fact that the growth of the Galaxy is followed under rather ideal conditions.

From the observational point of view, there seems to be clear consensus that the outer halo is very lumpy. This would imply that there is little room for a component predominantly formed via a dissipative collapse.

In the case of the inner stellar halo, the situation is much less clear. Recently, Morrison et al. (2008a) analyzed the distribution of angular momentum for a sample of nearby metal-poor halo stars with accurate velocities. These authors found a break in the properties of these stars depending on their angular momentum as shown in Fig. 15. The distribution of angular momentum is clearly not smooth, in particular for the higher halo (stars on highly inclined orbits), which suggests some amount of accretion in the build-up of this component.

Perhaps the most direct way of testing the amount of "dry" (i.e. dissipationless) accretion or mergers that have contributed to the stellar halo near the Sun, is to find the debris streams that are expected to be present. The granularity in the velocity distribution of stars should be measurable, if present, in large catalogs of halo stars $(>5,000$ stars) with very accurate kinematics (velocity errors $\leq 10 \mathrm{~km} \mathrm{~s}^{-1}$ ). The only processes by which these streams are expected to diffuse away are through collisions, or if the gravitational potential is (or has been) extremely chaotic. Galaxies are essentially 


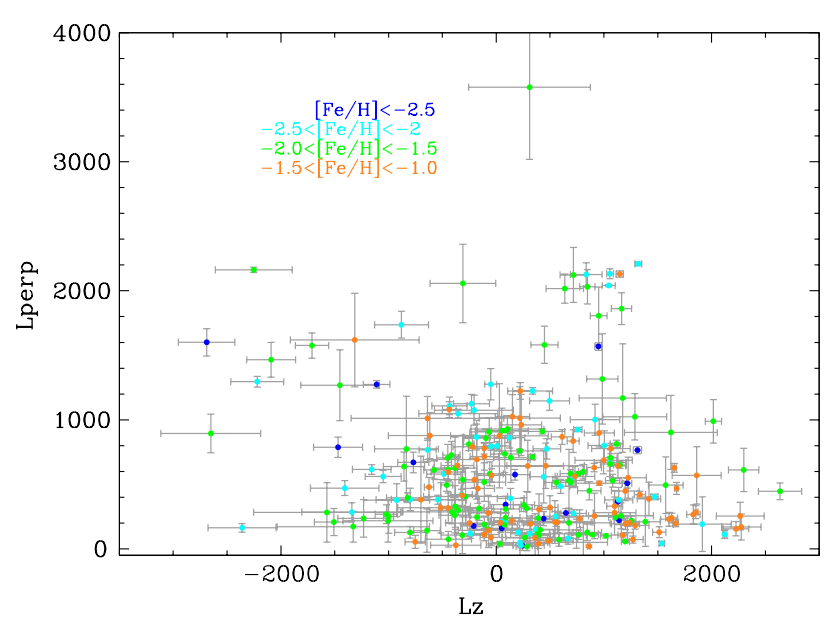

Fig. 15 Distribution of angular momentum for a sample of nearby halo stars. The color-coding indicates different metallicity ranges. Stars with orbits confined to the plane (Lperp $=\sqrt{L_{x}^{2}+L_{y}^{2}}<350 \mathrm{kpc}$ $\mathrm{km} \mathrm{s}^{-1}$ ) tend to have a net prograde motion (corresponding to $V_{\phi} \sim 50 \mathrm{~km} \mathrm{~s}^{-1}$ ), and their distribution is very smooth in this space. Stars with highly-inclined orbits (Lperp $>350 \mathrm{kpc} \mathrm{km} \mathrm{s}^{-1}$ ) have no net rotation and their distribution is quite lumpy, indicative of the presence of merger debris. It is also interesting to note that this "higher halo" is more metal-poor on average. (From Morrison et al. 2008a. Courtesy of Heather Morrison)

collisionless systems (Binney and Tremaine 1987), and the effect of dark matter substructure on the coherence of tidal streams has been shown to be very small (Johnston et al. 2002). Therefore one may only need to worry about the chaotic nature of the potential. First studies performed on fully cosmological dark matter simulations show evidence that the number of stellar streams expected near the Sun is the same as in steady-state models of the accretion of satellite galaxies onto a fixed gravitational potential (Helmi et al. 2003). The Galactic bar may also be a source of orbital diffusion. This triaxial potential is known to induce resonances amongst stars on low inclination orbits (Dehnen 2000; Famaey et al. 2005), and may well cause streams to become more diffuse in time, and hence harder to recover.

Another promising approach is that of chemical tagging, i.e. stars born within the same molecular cloud should have very similar chemical abundances as shown by De Silva et al. (2007). We have yet to understand how much scatter there can be in a galaxy that was accreted very early on, and in particular, if similar degrees of coherence in the abundances are expected for such (much larger) systems. For example, dwarf galaxies today show metallicity spreads of the order of 0.5 dex (Mateo 1998). Although one expects stars in a stream to come from a small region of phase-space, it is unclear how different their chemical abundances may be. For example, a satellite that has been disrupted only relatively recently will give rise to streams that are less chemically homogeneous than one whose streams are dynamically older. In the first case, the metallicity spread will likely reflect that of the whole galaxy, while in the second case it may reflect only that of a small portion of the system. In the limit of an infinite amount of time, the stars in a given location on a stream should have all been 
born within the same molecular cloud, and hence have the same exact abundances, provided diffusion processes are unimportant.

An accurate measurement of the ages of stars in the halo would yield unique insights. This would give a very robust test of the degree of dissipation involved in the formation of the stellar halo. For example, if the stars were formed predominantly during gas-rich mergers, the star formation history of the halo would have been largely bursty. In this case, each burst would be associated to each one of those gas-rich merger events. If on the other hand, the halo formed only via the accretion of a few objects containing some stars, each of these objects will have had a different star formation history, and hence a larger spread in ages might be expected. Note that in the limit in which gas-rich mergers have taken place successively on a very short timescale, such a model will be essentially indistinguishable from the original Eggen et al. (1962) proposal for the formation of the inner halo, even if the $\Lambda \mathrm{CDM}$ model is correct.

\section{Halos in other galaxies}

Stellar halos are generally such low surface brightness components of galaxies that detecting these beyond the Milky Way is a major challenge. For example, Morrison (1993) estimate the surface brightness of the Galactic halo at the solar radius to be $\Sigma_{V} \sim 27.7 \mathrm{mag} / \operatorname{arcsec}^{2}$.

Direct testimony of the difficulty of such an enterprise is given by M31. Its spheroid was long believed to be a simple extension of its bulge, since "metal-rich" stars were found even out to a radius of $30 \mathrm{kpc}$ following a de Vaucouleurs profile (e.g. Mould and Kristian 1986; Brown et al. 2003). However, Kalirai et al. (2006) and Chapman et al. (2006) recently discovered a metal-poor halo out to $160 \mathrm{kpc}$ that is similar to that of the Galaxy, both in terms of its metallicity, radial profile, as well as the amount of substructure. Our external point of view has allowed the study of this halo in a much more global fashion (Ibata et al. 2007), and has provided spectacular images as shown in Fig. 16. Just as for the Milky Way, in the halo of M31 we find a giant stream as well as a wealth of fainter substructures at surface brightness levels of $\sim 30 \mathrm{mag} / \mathrm{arcsec}^{2}$.

M33 seems to have a halo much like that of the Milky Way and M31, with a similar metallicity $[\mathrm{Fe} / \mathrm{H}] \sim-1.6$ dex, despite its smaller total mass (McConnachie et al. 2006). There is also some evidence that the Large Magellanic Cloud may have a stellar halo (Minniti et al. 2003). However, the origin of this halo is less clear because the LMC appears to have been tidally perturbed by the Milky Way (its disk is asymmetric and fairly thick as shown by van der Marel 2001; van der Marel et al. 2002). Such strong tides could give rise to a halo-like component (Mayer et al. 2001).

Beyond the Local Group, there have been a few studies of halos using surface brightness photometry. This work is very challenging because it requires characterizing a component several magnitudes below the sky level. A few halos have been detected in this way (e.g. NGC5907, NGC3115, see Morrison et al. 1994; Sackett et al. 1994) as well as various tidal features (Zheng et al. 1999; Pohlen et al. 2004). An interesting detection was obtained by Zibetti et al. (2004) who, stacking images of 1,047 edge-on disk galaxies, were able to measure a diffuse moderately flattened stellar halo, well described by a power law $r^{-3}$ density profile. 


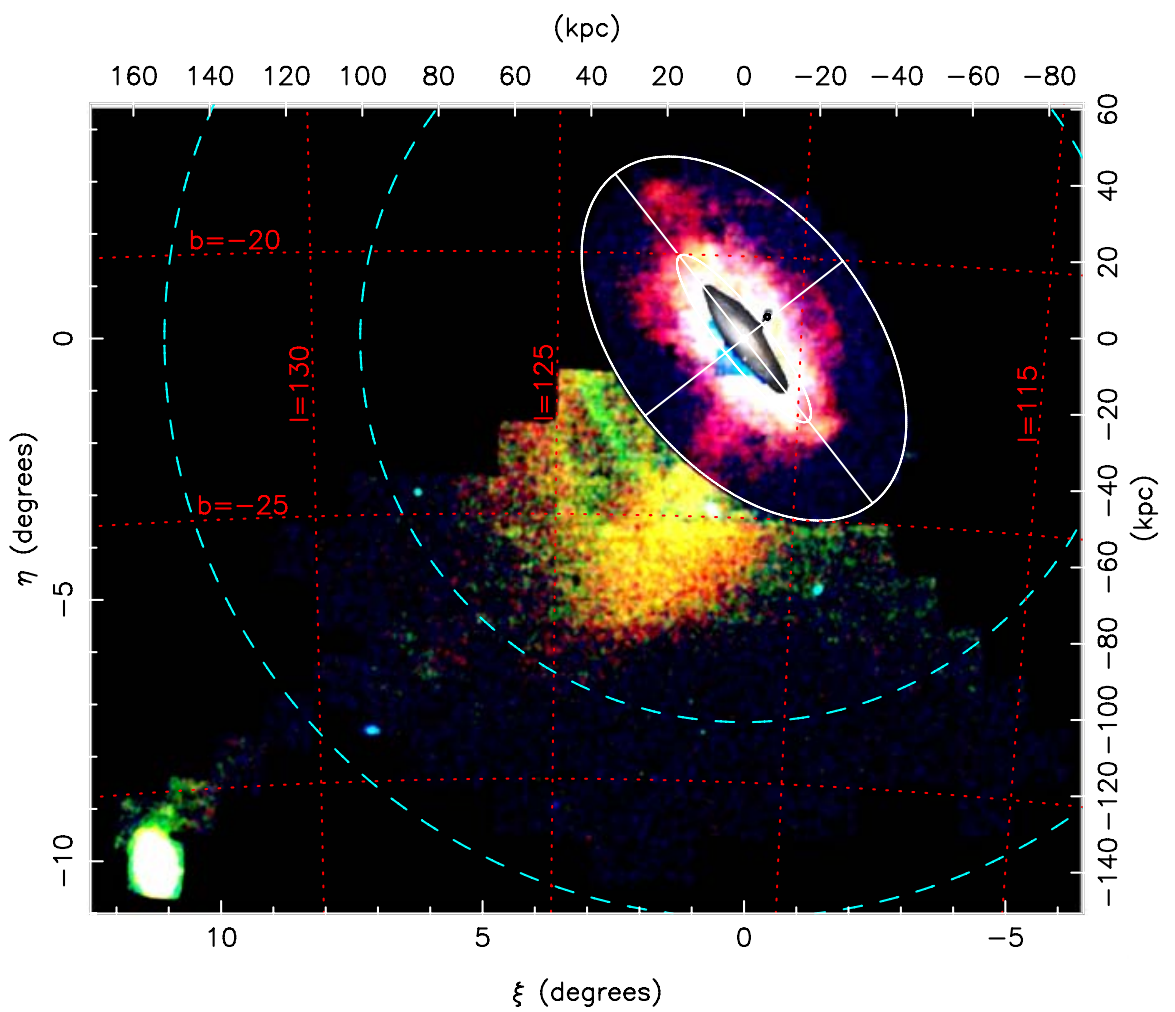

Fig. 16 View of the halo of M31. Red, green and blue show, respectively, stars with $-0.7<[\mathrm{Fe} / \mathrm{H}]<0$, $-1.7<[\mathrm{Fe} / \mathrm{H}]<-0.7$ and $-3.0<[\mathrm{Fe} / \mathrm{H}]<-1.7$. The differences in stellar populations between the Giant Stream and the several minor axis streams can be seen as striking differences in color. At the center there is a scaled image of the central of M31. (From Ibata et al. 2007. Courtesy of Rodrigo Ibata. Reproduced with permission of the AAS)

An alternative, possibly more powerful approach is to study the resolved stellar populations in the outskirts of (disk) galaxies, as frequently done in the Local Group. Using the Advanced Camera for Surveys (ACS) on HST Mouhcine et al. (2005b) and de Jong et al. (2007) have been able to resolve stars a few magnitudes below the tip of the red giant branch for a sample of nearby disk galaxies (located at distances between 4 and $14 \mathrm{Mpc}$ ). For example, Mouhcine et al. (2005b) find that the stellar halos are generally old, and typically more metal-poor than the main galaxy. These authors also find large variety in their properties. An example is NGC891, often referred to as the twin of the Milky Way, whose halo contains a much larger spread in stellar populations than is apparent for the Milky Way at a similar distance (Mouhcine et al. 2007). On the other hand, Mouhcine et al. (2005a) have suggested that there is a correlation between the stellar halo metallicity and the parent galaxy luminosity. Our Galaxy falls off this metallicity-luminosity relation by more than 1 dex, i.e. the Galactic stellar halo would be unusual, or too metal-poor (see also Hammer et al. 2007 for a similar statement).

Some caution is necessary when interpreting these observations, especially for the following two reasons. First, in the absence of spectroscopic measurements, the 
chemical properties of the stars cannot be determined reliably because of the wellknown age-metallicity degeneracy (a clear example of which is the dwarf galaxy Carina, Koch et al. 2006). Secondly, stellar halos can be very lumpy, particularly at very low surface brightness levels, as shown in Figs. 4 and 16. ACS/HST has a small field of view, implying that the results are quite sensitive to small-scale substructure. It is not unlikely, given what we know about the halos of the Milky Way and M31, that such a field would be dominated by just one stream such as for example the Sagittarius or the Giant stream around Andromeda. It may be misleading to correlate the properties of a dynamically young component such as the (outer) stellar halo, with the global properties of the parent galaxy, until a more global view of these halos is available (Ferguson 2007).

The avenue of mapping and resolving the stellar halos of nearby disk galaxies is clearly a very worthwhile goal, and one that should be pursued in the near future. The advent of Extremely Large Telescopes (ELTs) should allow wide-field surveys of the halos of nearby galaxies (also of earlier type), perhaps out to the Virgo cluster. A significant addendum would be spectroscopy, both to determine metallicities as well as the dynamics in the outskirts of these objects. In the hierarchical paradigm all galaxies should have experienced mergers, and most frequently minor mergers because most of the galaxies in the Universe are dwarfs. This implies that their debris should end up in a stellar halo, and so this component is expected to be ubiquitous. Therefore, unique insights into the assembly histories of galaxies as well as on their dynamics can be retrieved by studying their stellar halos.

\section{Epilogue}

The assembly process of a galaxy leaves imprints in the spatial, kinematical, age and chemical distribution of its stars. We have argued here that the Galactic halo should hold some of the best preserved fossils of the formation history of our Galaxy.

A stellar halo may well be the most common galaxy component and a natural outcome of the hierarchical build-up of galactic systems. For example, mergers are expected to deposit debris in a spheroidal like configuration. In the case of our Galaxy, both the halo field population, as well as the globular clusters (Côté et al. 2000) may have such an origin, as evidenced by e.g. the Sgr dwarf (Bellazzini et al. 2003; Geisler et al. 2007). Just like for stars, it is also possible that some fraction of the globular clusters formed in situ, perhaps during early gas-rich mergers that were presumably extremely common in the past (Kravtsov and Gnedin 2005). By studying the dynamical properties of the globular cluster population, and linking these to those of the field stars, such formation scenarios can be tested (e.g. Casetti-Dinescu et al. 2007).

At present it is unclear what fraction of the Galaxy's stellar halo has been built via dissipationless merging, and which by the gravitational collapse of one or more gas clouds (i.e. via a monolithic-like collapse or during gas rich mergers). There is significant evidence of substructure in the form of streams in the outer halo, but this is still at a rather qualitative level (although see Bell et al. 2007). It is also very likely that there is currently a bias towards detecting the highest surface brightness features, which presumably correspond to the most recent accretion events of relatively massive satellites. Deep wide-field photometric surveys should allow us to quantify the amount 
of substructure in the outer halo in an unbiased manner, especially if accompanied by spectroscopic surveys. Accurate photometry to enable quick and easy identification of suitable halo tracers are a must. Planned surveys such as VISTA, ${ }^{12}$ the OmegaCam on the VLT Survey Telescope, SkyMapper, PanStarrs ${ }^{13}$ and the Large Synoptic Survey Telescope (LSST) ${ }^{14}$ will significantly contribute to this enterprise. Wide-field imaging of nearby disk galaxies as ongoing with HST (de Jong et al. 2007), and as discussed for the ELTs, will allow us to establish the commonality of such merger events.

The dynamical timescales are much shorter in the inner regions of galaxies, which implies that halo substructures are no longer apparent in physical space, but become very prominent in velocity space. For example, a galaxy of the size of the Small Magellanic Cloud should have contributed 50-100 streams/cold moving groups near the Sun if accreted roughly $10 \mathrm{Gyr}$ ago (Helmi and White 1999). This implies that full phase-space information is absolutely vital to recover the $\sim 500$ streams predicted to be present in the inner stellar halo if this had been built up only via "dry" mergers. Current datasets are too small, or only have a limited amount of sufficiently accurate phase-space information to allow testing such a scenario. Of course this implies that it is still possible that the halo formed largely during a gas rich (merger) phase.

The definitive understanding of the structure and history of the Galactic stellar halo is likely to be established only via a "multi-dimensional" approach. Knowledge of the stars kinematics complemented with their chemical abundances and ages should allow us to address questions such as:

1. What fraction of the halo has been built via accretion? How does the importance of this process change with distance from the Galactic center?

2. When was the halo in place?

3. How many accreted objects have contributed to build up our Galaxy? Were these objects major contributors of cold gas, that later produced the disk? Is there an evolutionary link between the disk and halo?

4. What is the relation between the bulge and the stellar halo? The bulge is as old as the halo but more metal-rich, and pressumably built partly by a dynamical instability which led to the bar. Yet, a fraction of its stars may have the same origin as some halo stars.

5. What were the properties of the accreted objects? What were their masses, their star formation and chemical histories? How do they compare to the small galaxies we see around us today?

6. What is the dynamical history of our Galaxy? How did its mass and gravitational potential evolve in time?

The answers to these and many other questions will all be within reach in the coming decade, when large datasets with full phase-space and astrophysical information become available, i.e. in the era of Gaia.

Acknowledgments This review is dedicated to Manuel and Mariano. Without their support and their patience it would have never come to an end! I am grateful to Michael Perryman for suggesting this review,

\footnotetext{
12 http://www.vista.ac.uk.

13 http://pan-starrs.ifa.hawaii.edu/public/.

14 http://www.lsst.org/.
} 
and for giving me a deadline (to try) to meet. NOVA, and NWO through the VIDI program are acknowledged for financial support. Many friends and colleagues have contributed to this review with thoughts, inspiration, figures and feedback. All the figures are examples of this. I would like to thank them and especially mention here Giuseppina Battaglia, Paul Harding, Vanessa Hill, Mariano Méndez, Heather Morrison, Michael Perryman, Annie Robin, Laura Sales, Else Starkenburg and Eline Tolstoy.

Open Access This article is distributed under the terms of the Creative Commons Attribution Noncommercial License which permits any noncommercial use, distribution, and reproduction in any medium, provided the original author(s) and source are credited.

\section{References}

Abadi MG, Navarro JF, Steinmetz M, Eke VR (2003) ApJ 591:499

Abadi MG, Navarro JF, Steinmetz M (2006) MNRAS 365:747

Abel T, Bryan GL, Norman ML (2000) ApJ 540:39

Adelman-McCarthy JK et al (2007) ApJS 172:634

Alcock C et al (MACHO coll.) (2000b) ApJ 542:281

Arnould M, Goriely S, Takahashi K (2007) Phys Rep 450:97

Ashman KM, Zepf SE (1998) Globular cluster systems. Cambridge University Press, Cambridge

Bahcall JN, Soneira RM (1980) ApJS 44:73

Battaglia G et al (2005) MNRAS 364:433

Battaglia G et al (2006) MNRAS 370:1055

Barklem PS et al (2005) A\&A 439:129

Baumgardt H, Kroupa P, Parmentier G (2008) MNRAS 384:1231

Beers TC et al (2000) AJ 119:2866

Beers TC, Christlieb N (2005) ARA\&A 43:531

Beers TC et al (2006) Exploiting large surveys for Galactic astronomy. In: 26th meeting of the IAU, Joint Discussion 13, 22-23 August 2006, Prague, JD13, \#26, 13

Bekki K, Chiba M (2001) ApJ 558:666

Bell EF et al (2007) ArXiv e-prints, 706, arXiv:0706.0004

Bellazzini M, Ferraro FR, Ibata R (2003) AJ 125:188

Belokurov V, Evans NW, Irwin MJ, Hewett PC, Wilkinson MI (2006a) ApJ 637:L29

Belokurov V et al (2006b) ApJ 642:L137

Belokurov V et al (2007a) ApJ 654:897

Belokurov V et al (2007b) ApJ 657:L89

Belokurov V et al (2007c) ApJ 658:337

Benson AJ (2005) MNRAS 358:551

Bica E, Bonatto C, Barbuy B, Ortolani S (2006) A\&A 450:105

Binney J, May A (1986) MNRAS 218:743

Binney J, Merrifield M (1998) Galactic Astronomy, Ch. 3-5 Princeton University Press, Princeton

Binney J, Tremaine S (1987) Galactic Dynamics. Ch. 4 Princeton University Press, Princeton

Bland-Hawthorn J, Freeman K (2000) Science 287:79

Bromm V, Coppi PS, Larson RB (1999) ApJ 527:L5

Brook CB, Kawata D, Gibson BK, Flynn C (2003) ApJ 585:L125

Brook CB, Kawata D, Scannapieco E, Martel H, Gibson BK (2007) ApJ 661:10

Brown TM et al (2003) ApJ 592:L17

Bullock JS, Kravtsov AV, Weinberg DH (2000) ApJ 539:517

Bullock JS, Kravtsov AV, Weinberg DH (2001) ApJ 548:33

Bullock JS, Johnston KV (2005) ApJ 635:931

Carretta E, Gratton RG, Sneden C (2000) A\&A 356:238

Carney BW, Laird JB, Latham DW, Aguilar LA (1996) AJ 112:668

Carollo D et al (2007) Nature 450:1020

Casetti-Dinescu DI, Girard TM, Herrera D, van Altena WF, López CE, Castillo DJ (2007) AJ 134:195

Cayrel R et al (2001) Nature 409:691

Cayrel R et al (2004) A\&A 416:1117

Cescutti G, François P, Matteucci F, Cayrel R, Spite M (2006) A\&A 448:557 
Chaboyer B (1998) Phys Rep 307:23

Chapman SC et al (2006) ApJ 653:255

Chen B et al (2001) ApJ 553:184

Chiappini C, Matteucci F, Gratton R (1997) ApJ 477:765

Chiba M, Yoshii Y (1998) AJ 115:168

Chiba M, Beers TC (2000) AJ 119:2843

Chiba M, Beers TC (2001) ApJ 549:325

Christlieb N (2006) In: Stellar evolution at low metallicity: mass loss, explosions, cosmology. ASP conference series, vol 353. p 271

Clewley L et al (2005) MNRAS 362:349

Clewley L, Kinman TD (2006) MNRAS 371:L11

Cohen JG et al (2004) ApJ 612:1107

Côté P, Marzke RO, West MJ, Minniti D (2000) ApJ 533:869

De Angeli F et al (2005) AJ 130:116

Dehnen W (2000) AJ 119:800

De Lucia G, Helmi A (2008) ArXiv e-prints, 804, arXiv:0804.2465

De Silva GM et al (2007) AJ 133:1161

de Jong RS, Radburn-Smith DJ, Sick JN (2007) ArXiv e-prints 710, arXiv:0710.5511

Diemand J, Madau P, Moore B (2005) MNRAS 364:367

Digby AP, Hambly NC, Cooke JA, Reid IN, Cannon RD (2003) MNRAS 344:583

Dinescu DI, Girard TM, van Altena WF (1999) AJ 117:1792

Dohm-Palmer RC et al (2001) ApJ 555:L37

Dolphin AE, Weisz DR, Skillman ED, Holtzman JA (2005) ArXiv Astrophysics e-prints, arXiv:astro$\mathrm{ph} / 0506430$

Duffau S et al (2006) ApJ 636:L97

Eggen OJ, Lynden-Bell D, Sandage AR (1962) ApJ 136:748

Famaey B et al (2005) A\&A 430:165

Fellhauer M et al (2006) ApJ 651:167

Ferguson A (2007) In: From stars to galaxies: building the pieces to build up the Universe. ASP conference series, vol 374. p 239

Flynn C, Gould A, Bahcall JN (1996) ApJ 466:L55

Font AS, Johnston KV, Bullock JS, Robertson BE (2006) ApJ 646:886

François P et al (2007) A\&A 476:935

Frebel A et al (2006) ApJ 652:1585

Frebel A et al (2007) ApJ 660:L117

Freeman KC (1996) In: Formation of the Galactic halo. Inside and out. ASP conference series, vol 92. p 3

Freeman KC, Bland-Hawthorn J (2002) ARA\&A 40:487

Frenk CS, White SDM (1982) MNRAS 198:173

Fuchs B, Jahreiß H (1998) A\&A 329:81

Fulbright JP (2002) AJ 123:404

Gao L et al (2004) MNRAS 355:819

Geisler D, Wallerstein G, Smith VV, Casetti-Dinescu DI (2007) PASP 119:939

Gilmore G, Reid N (1983) MNRAS 202:1025

Gilmore G, Wyse RFG, Kuijken K (1989) ARA\&A 27:555

Gilmore G, Wyse RFG (1998) AJ 116:748

Gnedin NY, Kravtsov AV (2006) ApJ 645:1054

Gómez-Flechoso MA, Fux R, Martinet L (1999) A\&A 347:77

Goriely S, Siess L (2005) In: From lithium to uranium: elemental tracers of early cosmic evolution. IAU symposium, vol 228. p 451

Gould A, Flynn C, Bahcall JN (1998) ApJ 503:798

Gould A (2003) ApJ 592:L63

Grebel EK (2000) In: Star formation from the small to the large scale. ESA SP, vol 445. p 87

Grillmair CJ, Freeman KC, Irwin M, Quinn PJ (1995) AJ 109:2553

Grillmair CJ (2006a) ApJ 651:L29

Grillmair CJ (2006b) ApJ 645:L37

Grillmair CJ, Johnson R (2006) ApJ 639:L17

Hambly NC et al (2001) MNRAS 326:1279

Hammer F, Puech M, Chemin L, Flores H, Lehnert MD (2007) ApJ 662:322 
Hansen BMS, Liebert J (2003) ARA\&A 41:465

Harding P et al (2001) AJ 122:1397

Harris WE (1976) AJ 81:1095

Hartwick FDA (1987) NATO ASIC Proc. 207: The Galaxy, 281

Hartwick FDA (2000) AJ 119:2248

Heger A, Woosley SE (2002) ApJ 567:532

Helmi A, White SDM (1999) MNRAS 307:495

Helmi A, White SDM, de Zeeuw PT, Zhao H (1999) Nature 402:53

Helmi A, de Zeeuw PT (2000) MNRAS 319:657

Helmi A, White SDM (2001) MNRAS 323:529

Helmi A, White SDM, Springel V (2002) Phys Rev D 66:063502

Helmi A, White SDM, Springel V (2003) MNRAS 339:834

Helmi A, Navarro JF, Meza A, Steinmetz M, Eke VR (2003) ApJ 592:L25

Helmi A (2004a) MNRAS 351:643

Helmi A (2004b) ApJ 610:L97

Helmi A et al (2006) ApJ 651:L121

Hill V et al (2002) A\&A 387:560

Hill $\mathrm{V}$ et al (2008) (in preparation)

Ibata R, Gilmore G, Irwin MJ (1994) Nature 370:194

Ibata R, Irwin M, Lewis GF, Stolte A (2001) ApJ 547:L133

Ibata R, Irwin MJ, Lewis GF, Ferguson AMN, Tanvir N (2003) MNRAS 340:L21

Ibata R et al (2007) ApJ 671:1591

Ivans II et al (2003) ApJ 592:906

Ivezić Ž et al (2000) AJ 120:963

Johnston KV, Hernquist L, Bolte M (1996) ApJ 465:278

Johnston KV, Majewski SR, Siegel MH, Reid IN, Kunkel WE (1999) AJ 118:1719

Johnston KV, Spergel DN, Haydn C (2002) ApJ 570:656

Johnston KV, Law DR, Majewski SR (2005) ApJ 619:800

Juric M et al (2008) ApJ 673:864

Kalirai JS et al (2006) ApJ 648:389

Kang X, Mao S, Gao L, Jing YP (2005) A\&A 437:383

Kapteyn JC, van Rhijn PJ (1920) ApJ 52:23

Kauffmann G, White SDM, Guiderdoni B (1993) MNRAS 264:201

Kepley AA et al (2007) AJ 134:1579

Kinman TD, Wirtanen CA, Janes KA (1966) ApJS 13:379

Kinman TD, Cacciari C, Bragaglia A, Buzzoni A, Spagna A (2007) MNRAS 375:1381

Klypin A, Kravtsov AV, Valenzuela O, Prada F (1999) ApJ 522:82

Klypin A, Zhao H, Somerville RS (2002) ApJ 573:597

Koch A et al (2006) AJ 131:895

Koposov S et al (2007) ArXiv e-prints, 706, arXiv:0706.2687

Kravtsov AV, Gnedin OY, Klypin AA (2004) ApJ 609:482

Kravtsov AV, Gnedin OY (2005) ApJ 623:650

Kroupa P, Theis C, Boily M (2005) A\&A 431:517

Layden AC, Hanson RB, Hawley SL, Klemola AR, Hanley CJ (1996) AJ 112:2110

Layden AC (1998) In: Galactic halos. ASP conference series, vol 136. p 14

Layden AC, Sarajedini A (2000) AJ 119:1760

Law DR, Johnston KV, Majewski SR (2005) ApJ 619:807

Lee YW et al (1999) Nature 402:55

Lee Y-W, Gim HB, Casetti-Dinescu DI (2007) ApJ 661:L49

Lemon DJ, Wyse RFG, Liske J, Driver SP, Horne K (2004) MNRAS 347:1043

Letarte B et al (2006) A\&A 453:547

Letarte B (2007) PhD thesis. University of Groningen, The Netherlands

Li YS, Helmi A (2008) MNRAS 385:1365

Libeskind $\mathrm{N}$ et al (2005) MNRAS 363:146

Lindegren L (2005) In: The three-dimensional Universe with Gaia. ESA SP, vol 576. p 29

Lucatello S, Gratton RG, Beers TC, Carretta E (2005) ApJ 625:833

Lynden-Bell D, Lynden-Bell RM (1995) MNRAS 275:429

Mackey AD, Gilmore GF (2004) MNRAS 355:504 
Mac Low M-M, Ferrara A (1999) ApJ 513:142

Majewski SR (1993) ARA\&A 31:575

Majewski SR, Munn JA, Hawley SL (1996) ApJ 459:L73

Majewski SR et al (1999) AJ 118:1709

Majewski SR, Skrutskie MF, Weinberg MD, Ostheimer JC (2003) ApJ 599:1082

Martin NF et al (2004) MNRAS 348:12

Martin NF, Ibata RA, Chapman SC, Irwin M, Lewis GF (2007) MNRAS 380:281

Martínez-Delgado D, Aparicio A, Gómez-Flechoso MÁ, Carrera R (2001) ApJ 549:L199

Martínez-Delgado D, Gómez-Flechoso MÁ, Aparicio A, Carrera R (2004) ApJ 601:242

Martínez-Delgado D et al (2005) ApJ 633:205

Mateo ML (1998) ARA\&A 36:435

Mateo M, Olszewski EW, Morrison HL (1998) ApJ 508:L55

Matteucci F (2001) Astrophysics and Space Science Library 253

Matteucci F, Recchi S (2001) ApJ 558:351

Mayer L et al (2001) ApJ 559:754

McConnachie AW et al (2006) ApJ 647:L25

McWilliam A (1997) ARA\&A 35:503

Metz M, Kroupa P, Jerjen H (2007) MNRAS 374:1125

Minniti D et al (2003) Science 301:1508

Moitinho A, Vázquez RA, Carraro G, Baume G, Giorgi EE, Lyra W (2006) MNRAS 368:L77

Momany Y et al (2004) A\&A 421:L29

Momany Y et al (2006) A\&A 451:515

Moore B et al (1999) ApJ 524:L19

Moore B (2001) In: 20th Texas symposium on relativistic astrophysics. AIP conference proceedings, vol 586. p 73

Moore B, Diemand J, Madau P, Zemp M, Stadel J (2006) MNRAS 368:563

Morrison HL (1993) AJ 106:578

Morrison HL, Boroson TA, Harding P (1994) AJ 108:1191

Morrison HL et al (2000) AJ 119:2254

Morrison HL et al (2008a) ArXiv e-prints, 804, arXiv:0804.2448

Morrison $\mathrm{H}$ et al (SDSS) (2008b) (in preparation)

Mouhcine M, Ferguson HC, Rich RM, Brown TM, Smith TE (2005) ApJ 633:821

Mouhcine M, Rich RM, Ferguson HC, Brown TM, Smith TE (2005) ApJ 633:828

Mouhcine M, Rejkuba M, Ibata R (2007) MNRAS 381:873

Mould J, Kristian J (1986) ApJ 305:591

Navarro JF, Abadi M, Steinmetz M (2004) ApJ 613:L41

Navarro JF, Frenk CS, White SDM (1996) ApJ 462:563

Newberg HJ et al (2002) ApJ 569:245

Newberg HJ, Yanny B (2006) J Phys Conf Series 47:195

Newberg HJ et al (2007) ApJ 668:221

Nissen PE, Gustafsson B, Edvardsson B, Gilmore G (1994) A\&A 285:440

Nissen PE, Schuster WJ (1997) A\&A 326:751

Odenkirchen M et al (2001) ApJ 548:L165

Paczynski B (1986) ApJ 304:1

Peñarrubia J et al (2005) ApJ 626:128

Perryman MAC et al (2001) A\&A 369:339

Phleps S, Drepper S, Meisenheimer K, Fuchs B (2005) A\&A 443:929

Piatek S et al (2007) AJ 133:818

Piotto G et al (2007) ApJ 661:L53

Pohlen M et al (2004) In: Satellites and tidal streams. ASP conference series, vol 327. p 288

Preston GW, Shectman SA, Beers TC (1991) ApJ 375:121

Quinn PJ (1984) ApJ 279:596

Ratnatunga KU, Freeman KC (1985) ApJ 291:260

Robin AC, Reylé C, Crézé M (2000) A\&A 359:103

Robin AC et al (2007) ApJS 172:545

Rocha-Pinto HJ, Majewski SR, Skrutskie MF, Crane JD, Patterson RJ (2004) ApJ 615:732

Ryan SG, Norris JE (1991) AJ 101:1865

Sackett PD, Morrison HL, Harding P, Boroson TA (1994) Nature 370:441 
Sales LV, Navarro JF, Abadi MG, Steinmetz M (2007) MNRAS 379:1464

Sales LV et al (2008) (in preparation)

Salvadori S, Schneider R, Ferrara A (2007) MNRAS 381:647

Salvadori S, Ferrara A, Schneider R (2008) ArXiv e-prints, 802, arXiv:0802.0462

Samland M, Gerhard OE (2003) A\&A 399:961

Sarajedini A, Chaboyer B, Demarque P (1997) PASP 109:1321

Schmidt M (1956) Bull Astron Inst Netherlands 13:15

Schneider R, Ferrara A, Natarajan P, Omukai K (2002) ApJ 571:30

Seabroke GM et al (2008) MNRAS 384:11

Searle L, Zinn R (1978) ApJ 225:357

Shapley H (1918) PASP 30:42

Shetrone M et al (2001) ApJ 548:592

Shetrone M et al (2003) AJ 125:707

Siegel MH, Majewski SR, Reid IN, Thompson IB (2002) ApJ 578:151

Siegel MH et al (2007) ApJ 667:L57

Silk J, Langer M (2006) MNRAS 371:444

Simon JD, Geha M (2007) ApJ 670:313

Sirko E et al (2004) AJ 127:914

Smith MC et al (2007) MNRAS 379:755

Sneden C et al (1996) ApJ 467:819

Sommer-Larsen J, Christensen PR (1989) MNRAS 239:441

Sommer-Larsen J, Beers TC, Flynn C, Wilhelm R, Christensen PR (1997) ApJ 481:775

Somerville RS, Primack JR (1999) MNRAS 310:1087

Spergel DN, Steinhardt PJ (2000) Physical Review Letters 84:3760

Springel V, Frenk CS, White SDM (2006) Nature 440:1137

Starkenburg E et al (2008) (in preparation)

Steinmetz M et al (2006) AJ 132:1645

Steinmetz M, Muller E (1995) MNRAS 276:549

Stetson PB, Vandenberg DA, Bolte M (1996) PASP 108:560

Stetson PB, Hesser JE, Smecker-Hane TA (1998) PASP 110:533

Stoehr F, White SDM, Tormen G, Springel V (2002) MNRAS 335:L84

Suntzeff NB, Kinman TD, Kraft RP (1991) ApJ 367:528

Tisserand et al. (EROS coll) (2007) A\&A 469:387

Tolstoy E et al (2003) AJ 125:707

Tolstoy E et al (2006) The Messenger 123:33

Torres S, García-Berro E, Isern J (1998) ApJ 508:L71

Travaglio C et al (2004) ApJ 601:864

Tumlinson J (2007) ApJ 665:1361

Umeda H, Nomoto K (2005) ApJ 619:427

Unavane M, Wyse RFG, Gilmore G (1996) MNRAS 278:727

van der Marel RP (2001) AJ 122:1827

van der Marel RP, Alves DR, Hardy E, Suntzeff NB (2002) AJ 124:2639

Venn KA et al (2004) AJ 128:1177

Vivas AK et al (2001). ApJ 554:L33

Vivas AK et al (2004) AJ 127:1158

Wheeler JC, Sneden C, Truran JW Jr. (1989) ARA\&A 27:279

White SDM (1985) ApJ 294:L99

White SDM, Frenk CS (1991) ApJ 379:52

Wilkinson MI, Evans NW (1999) MNRAS 310:645

Yanny B et al (2000) ApJ 540:825

Yanny B et al (2003) ApJ 588:824

Xu Y, Deng LC, Hu JY (2006) MNRAS 368:1811

Xu Y, Deng LC, Hu JY (2007) MNRAS 379:1373

Zentner AR et al (2005) ApJ 629:219

Zheng Z et al (1999) AJ 117:2757

Zibetti S, White SDM, Brinkmann J (2004) MNRAS 347:556

Zinn R (1993) In: The globular cluster-galaxy connection. ASP conference series, vol 48. p 38 